\title{
The Effect of Concurrent Multi-priority Data Streams on the MAC Layer Performance of IEEE 802.11p and C-V2X Mode 4
}

\author{
Geeth P. Wijesiri N.B.A, Member, IEEE, Jussi Haapola, Member, IEEE, and Tharaka Samarasinghe, Senior \\ Member, IEEE
}

\begin{abstract}
Supporting parallel multi-priority data streams is vital for maintaining the quality of service $(\mathrm{QoS})$ in vehicle-toeverything (V2X) communication. Hence, the European telecommunications standard institute (ETSI) has defined four packet types, with varying priority levels, to be used as broadcast packets in such communication. This paper studies the medium access control (MAC) layer performance of IEEE 802.11p and cellular-V2X (C-V2X) Mode 4 using discrete-time Markov chain (DTMC) based models, while considering parallel multi-priority data streams. The overall model consists of four queue models with their respective traffic generators, which are appropriately linked with the DTMCs modeling the MAC layer operations of IEEE 802.11p and C-V2X Mode 4. Closed-form solutions for the steady-state probabilities of the models are obtained, which are then utilized to derive expressions for key performance indicators at the MAC layer. Numerical results are provided to draw insights on the MAC layer performance of the two technologies. IEEE 802.11p is comparatively superior in average delay, and at maintaining fairness among multi-priority data streams, whereas C-V2X Mode 4 exhibits better collision resolution, which leads to its higher throughput. The paper also includes design insights on possible performance enhancements for future releases.
\end{abstract}

Index Terms-Cooperative environmental notification messages, C-V2X Mode 4, decentralized environmental notification messages, discrete-time Markov chain, IEEE 802.11p, medium access control, vehicle-to-vehicle communication.

\section{INTRODUCTION}

\section{A. Background and Motivation}

O UPPORTING multi-priority data streams plays a pivotal role in satisfying the stringent quality of service (QoS) requirements set for vehicle-to-everything (V2X) communications. Against this backdrop, the European telecom-

G. P. Wijesiri N.B.A is with the Department of Electronic and Telecommunication Engineering, University of Moratuwa, Sri Lanka, and the Department of Electrical and Information Engineering, University of Ruhuna, Sri Lanka (e-mail: geeth@eie.ruh.ac.lk).

J. Haapola is with the Center for Wireless Communications, University of Oulu, Finland (e-mail: jussi.haapola@oulu.fi).

T. Samarasinghe is with the Department of Electronic and Telecommunication Engineering, University of Moratuwa, Sri Lanka, and the Department of Electronic and Electrical Engineering, University of Melbourne, Australia (e-mail: tharakas@uom.lk).

This research has partially received funding from the European Union's Horizon 2020 research and innovation programme under grant agreement No 857031 (project 5G!Drones), the Academy of Finland 6Genesis Flagship (grant 318927) and the AHEAD/RA3/RIC/MRT/ITS-Multidisciplinary Transport Development Project of the University of Moratuwa under World Bank grant 6026-LK/8743-LK.

The material in this paper was presented in part at the IEEE Global Communications Conference, Taipei, Taiwan, Dec. 2020. munications standard institute (ETSI) intelligent transportation system (ITS) G5 [1] has defined four traffic classes that utilize different broadcast packets, namely, decentralized environmental notification messages (DENM), high priority DENM (HPD), cooperative awareness messages (CAM), and multi-hop DENM (MHD), for V2X communication. Each packet type has its own frequency of generation, number of repetitions, latency constraints, and a level of priority. The different packet types allow the two enabling technologies of V2X, which are IEEE 802.11p and cellular-V2X (C-V2X), to cater a multitude of ITS applications having varying QoS requirements.

The periodic transmission of CAM packets governs information sharing, such as position, dynamics and attributes, and facilitates cooperative awareness [2]. HPD, DENM, and MHD, on the other hand, are event-driven messages utilized to communicate random events such as human or environment initiated disturbances [3]. DENM are used in comparatively less critical scenarios, such as roadwork warnings and safety function out of normal condition warnings, compared to HPD, which are utilized for highly critical scenarios such as emergency electronic brake light warnings. MHD are the relayed DENM packets from other vehicles, and they get the lowest priority upon transmission because a vehicle gives more priority for its own packets. The parallel operation of multi-priority broadcast data streams and the strict latency and reliability constraints in V2X communications make the medium access control (MAC) layer operation of the two enabling technologies of $\mathrm{V} 2 \mathrm{X}$ crucial, which triggers our motivation for this study.

IEEE 802.11p [4] is the first WiFi-based standard designed explicitly for vehicular communications. The MAC layer operation of IEEE 802.11p stems from the well-known carrier sense multiple access with collision avoidance (CSMA/CA) technique. The enhanced distributed channel access (EDCA) mechanism [5] was introduced for IEEE 802.11p to allow the vehicle to accommodate differential QoS levels through four access categories (ACs), namely voice $A C_{v o}$, video $A C_{v i}$, best-effort $A C_{b e}$, and background $A C_{b k}$. These ACs have different parameters for channel contention such that the QoS requirements of multiple data traffic classes can be met. Each $\mathrm{AC}$ also has an associated packet type, with a defined priority level [1]. In this paper, we focus on the European version of $802.11 \mathrm{p}$ termed as the ETSI ITS-G5 IEEE 802.11p. CV2X Mode 4 introduced in 3GPP Release 14, that has similar 
capabilities to IEEE $802.11 \mathrm{p}$, can be considered to be the main competing technology [6], [7]. C-V2X Mode 4 uses the distributed sensing mechanism called semi-persistent scheduling (SPS) [8] to forecast and schedule free radio resources. However, there is no special mechanism to support multipriority data streams in $\mathrm{C}-\mathrm{V} 2 \mathrm{X}$ Mode 4 similar to EDCA in IEEE 802.11p. Differential QoS is achieved by giving preference to higher priority packets, upon a transmission opportunity. Our paper primarily focuses on utilizing discretetime Markov chains (DTMCs) for analytical modeling of the MAC layer operations of ETSI ITS-G5 IEEE 802.11p and CV2X Mode 4, in the presence of parallel multi-priority data streams.

\section{B. Related Works and Novel Contributions}

Several research works that focus on the MAC layer performance of IEEE $802.11 \mathrm{p}$ in the presence of parallel multipriority data streams can be identified in the literature [9]-[13]. The initial work on the MAC layer performance modeling of the IEEE 802.11p EDCA mechanism considered only a selective subset of the four ACs [9]-[11]. Subsequently, the authors of [12] and [13] considered the parallel operation of all four ACs in their DTMC-based modeling, and can be considered to be closely related to our work. Our model firstly improves slight inconsistencies of [12] and [13] with the standard, in terms of broadcast traffic. However, the main novelty in our work is the increased modeling granularity. This allows us to study the MAC layer operations for each aSlotTime, which is the smallest time unit in the IEEE 802.11p standard, similar to [14]. The increased granularity makes our model more complex, but in turn, more accurate than [12] and [13]. We precisely model the waiting times of different ACs before resuming channel contention, enabling us to correctly capture the effect of prioritization among the ACs, which are not done in [12] and [13]. An AC bearing a higher priority can initiate transmission during the long waiting period of an AC with lower priority. Transmission delays are also calculated more accurately than in [12] and [13] by taking the payload size into account.

Performance analysis of C-V2X Mode 4 has become an active research area recently. The related works can be mainly categorized as simulation [15], [16] and analytical modeling based [14], [17]-[19] studies. The authors of [15] present the first open-source simulator for C-V2X Mode 4 by utilizing the NS3 software, and study the performance of the technology. A similar study using the VEINS open-source framework is presented in [16]. The first analytical model for the MAC layer operations of C-V2X Mode 4 is proposed in [17]. The paper considers a PHY layer model to capture the effect of distance between the vehicles, and the MAC layer operation is handled by the SPS algorithm [8]. The authors then obtain analytical expressions for several performance measures at the MAC layer as a function of the distance between the communicating nodes. In [18], the authors compare the MAC layer performance of C-V2X Mode 4 with IEEE $802.11 \mathrm{p}$ for the transmission of periodic and aperiodic messages, having both fixed and variable sizes. The paper highlights certain inefficiencies of the SPS algorithm when transmitting aperiodic messages with variable sizes. The first DTMC-based analytical model for the MAC layer operations of C-V2X Mode 4 is proposed in [14], [19]. The overall model in our paper stems on this model, while additionally capturing the effect of parallel multi-priority data streams. The model also considers the requirement of adjusting the selection window size based on the number of vehicles, to facilitate the radio resource selection process according to the SPS algorithm. To the best of our knowledge, this is the first paper that focuses on the MAC layer performance of C-V2X Mode 4 in the presence of parallel multi-priority data streams.

The main contributions of our paper can be further summarized as follows:

- Modeling multi-priority packets: We model the generation of multi-priority data packets, namely HPD, DENM, CAM, and MHD, by utilizing four DTMCs. The separate DTMCs provide us the flexibility of altering the traffic arrival patterns of the individual packet types, and create a more realistic V2X communication scenario compared to the simpler models with predefined traffic rates used in [12] and [13]. We use four more DTMCs to model the device-level packet queues of the generated packet types, and each generator model is appropriately coupled with its queue model.

- Modeling the MAC layer operations: We then model the MAC layer operations of each V2X enabling technology using DTMCs. These models are appropriately coupled with the generator and queue models while taking priority levels among data streams into consideration. The priority management makes this paper significantly different from [14], in terms of the overall models, as well as the results and the drawn insights. Since [14] neglects the priority levels of the packets, the generated packets are transmitted in the order of generation.

- Performance analysis: We utilize the closed-form steady-state probabilities of the DTMCs to derive expressions for the average delay, collision probability, throughput, and the channel utilization.

- Comparison of the two technologies: We then present an application of the models. We first highlight the importance of taking all four parallel data streams into consideration when studying the MAC layer performance of the two technologies, by comparing with a scenario where only a subset of the packet types is utilized for communication. We compare the two technologies using the derived performance measures while providing insights on the synchronous operation of multi-priority data streams. In particular, IEEE 802.11p is superior in average delay, but exhibits a higher number of collisions compared to C-V2X Mode 4. Also, it can be seen that IEEE $802.11 p$ treats multi-priority data streams more fairly compared to its counterpart, thanks to its inbuilt EDCA mechanism.

- Insights on performance enhancements: The numerical evaluations are used to draw insights on how the performance of each technology can be enhanced in the presence of parallel multi-priority data streams. We also 
present comparisons with similar results obtained according to the models in [14] to highlight the significance of priority management in the MAC layer performance. These insights can contribute in the evolution of these two technologies into new radio V2X (NR-V2X) and IEEE 802.11bd [20], [21].

The paper is organized as follows. The DTMCs and the evaluation of the dependencies among the DTMCs are presented in Section II and Section III, respectively. The performance analysis follows in Section IV. Section V consists of the numerical results and discussion, and Section VI concludes the paper.

\section{Analytical Models}

This section presents the DTMC-based analytical models utilized to study the effect of concurrent multi-priority data streams on the MAC layer performance of IEEE 802.11p and C-V2X Mode 4. A DTMC is a stochastic model that describes a random process utilizing a discrete set of states in discrete-time, and follows the Markov property, i.e., the future evolution of the process depends only on the present state. A state change in a DTMC is called a transition, and the associated probability is called the transition probability. DTMCs have been studied for decades to model a wide range of real-world applications [22]. There are two main reasons for using DTMC for this study. Firstly, DTMC-based modeling facilitates improved granularity in the models. This enables us to precisely model the MAC layer operations in discrete time. Secondly, the memoryless property of DTMCs makes the analysis mathematically tractable. All DTMCs in this paper are aperiodic and irreducible, thus they are ergodic. Hence, a steady-state distribution for each model exists [23]. The models cater for both periodic and event-driven traffic (non-saturated transmission conditions), but do not account for phenomena at the physical (PHY) layer such as mobility, distance, and speed. We consider a vehicular network of $N$ vehicles, and we use $v$ to denote the target vehicle.

The overall models for IEEE 802.11p and C-V2X Mode 4 are presented in Figs. 1a and 1b, respectively. In our notations, we use subscripts $H, D, C$, and $M$ to differentiate between HPD, DENM, CAM, and MHD, respectively, and superscripts $11 p$ and $v 2 x$ to differentiate between IEEE 802.11p and CV2X Mode 4. The overall models consist of multiple interrelated DTMCs. The parameters that introduce dependencies among the DTMCs (e.g., $\alpha_{l}^{k}, \alpha_{1, l}^{k}, \beta_{l}^{k}, P_{q e, l}^{k}, P_{t, l}^{11 p}, P_{t}^{v 2 x}$, $P_{a r r, l}^{11 p}, P_{q e}^{v 2 x}, P_{a r r}^{v 2 x}$, for $\left.l \in\{H, D, C, M\}, k \in\{11 p, v 2 x\}\right)$, are introduced formally later in the section, while discussing the related DTMCs. Firstly, we use a common structure of four DTMCs to model the generation of HPD, DENM, CAM, and MHD packets, for both technologies. We refer to them as packet generators, and the priority order of serving these packets is as follows: HPD $>$ DENM $>$ CAM $>$ MHD. The common structure for packet generation facilitates a fair performance comparison of the two technologies. Secondly, we use four DTMCs to model the device-level packet queues of the generated packet types. The queue models introduced for the two technologies are slightly different from one another.
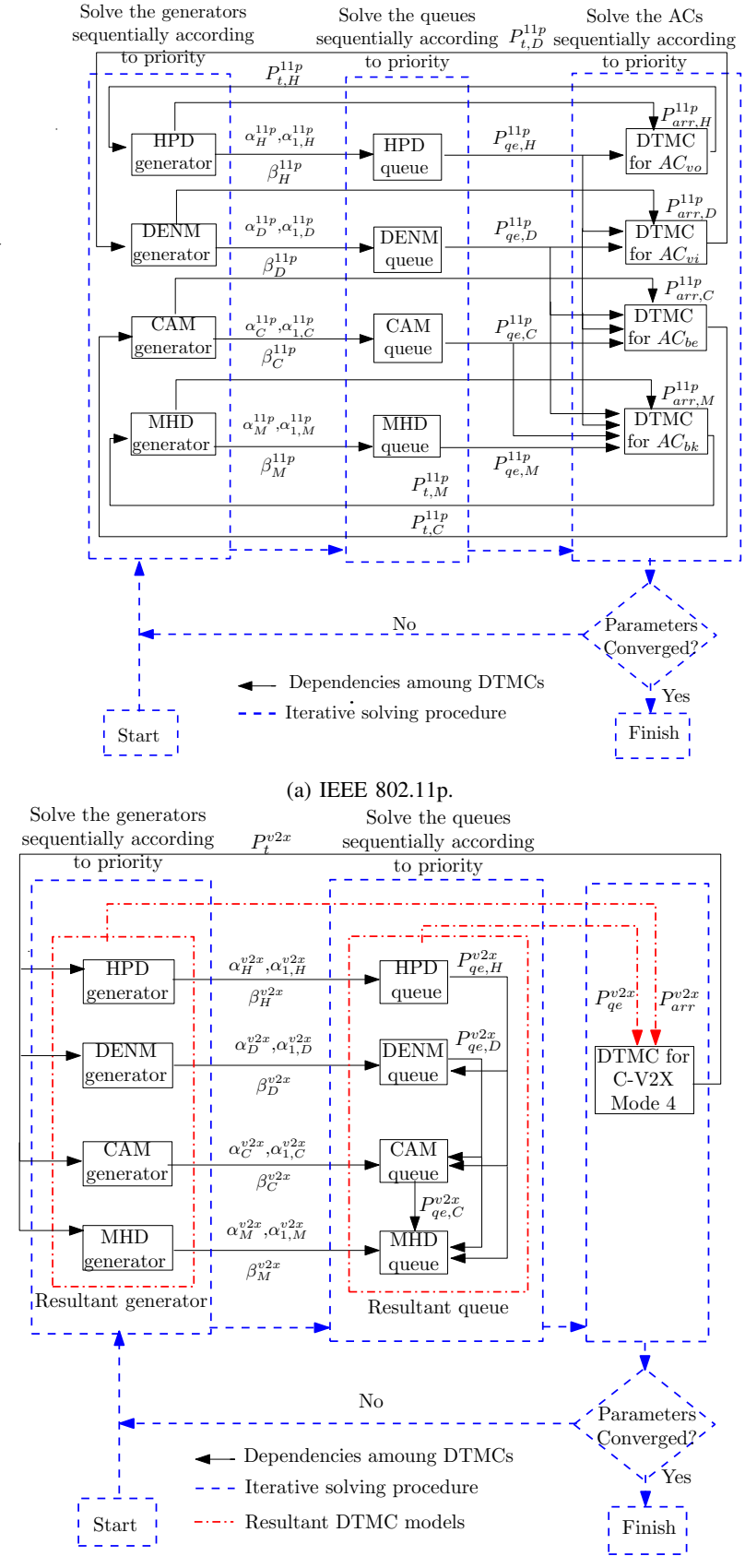

(b) C-V2X Mode 4 .

Figure 1: Flowchart illustrating the overall models.

The overall models differ significantly in the third stage that consists of the DTMCs modeling the MAC layer operations. IEEE 802.11p allows the vehicle to accommodate differential QoS levels through four ACs, namely, voice $A C_{v o}$ utilized for HPD packets, video $A C_{v i}$ utilized for DENM packets, besteffort $A C_{b e}$ utilized for CAM packets, and background $A C_{b k}$ utilized for MHD packets [1]. Thus, the MAC layer operations of IEEE 802.11p are modeled using four separate DTMCs (one for each $\mathrm{AC}$ ), as shown in Fig. 1a, while incorporating the effect of priority. On the other hand, the MAC layer operation of C-V2X Mode 4 does not directly consider priority levels upon transmission. Therefore, a single DTMC is used to model the MAC layer operation, while considering the resultant effect of the generators and the queues, as shown in Fig. 1b. The priority management is incorporated in the queues, which leads 
to the aforementioned difference in the queue models of the two technologies. Figs. 1a and $1 \mathrm{~b}$ also illustrate the iterative solving procedure of these models, which we explain in detail in Section V. Next, we present further details on the individual DTMCs.

\section{A. Packet Generators}

HPD, DENM, and MHD are all randomly generated packets, thus we model their arrivals through Poisson arrival processes of intensity $\lambda_{m}$, where $m \in\{H, D, M\}$. Due to their critical nature, HPD and DENM packets are repeated at fixed periods of $T_{H}$ and $T_{D}$, respectively, for added reliability [24]. This means the HPD and DENM generators capture two distinct packet types: a Poisson-based triggered generation and the subsequent fixed-period repeat packet generation. Upon a trigger, an HPD packet is transmitted $K_{H}$ times, and a DENM packet is transmitted $K_{D}$ times, periodically. MHD packets are generated by neighboring vehicles, and supposed to be forwarded by vehicle $v$ with the lowest priority. Hence, these packets are not repeated, and the number of repetitions of an MHD packet $K_{M}$ is set at one. CAM packets, on the other hand, have a periodic packet arrival pattern, and the interarrival time $T_{C}$ takes a value between $100 \mathrm{~ms}$ and $1000 \mathrm{~ms}$ [2].

The four generator models are illustrated using a single figure (Fig. 2). DTMCs for the generation of CAM and DENM packets are introduced in [14], and in this paper, we have included two more variants of DENM, which are HPD and MHD. All four models are presented in this paper for completeness and consistency in notations. The parts common to all generators are represented using solid lines, and different line styles are used to highlight the parts specific to a certain generator appropriately. The three event-driven generators consist of state (Idle) to account for the periods with no generated packets. Note that since $K_{M}=1$, the MHD generator simplifies into a two state DTMC model which consists of (idle) and $(t x, 0)$ states. The triggered generation of MHD packets is represented by the transition from state (idle) to $(t x, 0)$ with probability $1-e^{-\lambda_{M} \tilde{T}}$, where $\tilde{T}$ is aSlotTime for IEEE 802.11p, and subframe for C-V2X Mode 4. State $(t x, 0)$ represents packet generation, and since $K_{M}=1$, the transition back to (Idle) happens with probability 1 .

The other three packet types (HPD, DENM, and CAM) share similarities with regards to the periodicity. In these generators, states $(i, 0), i \in\left\{t x, t x^{\prime}\right\}$, represent the packet generation, where $t x$ and $t x^{\prime}$ are used to represent whether the previously generated packet has been transmitted, or not, respectively. The waiting time between the generation of two packets, denoted by $T_{n} \mathrm{~ms}, n \in\{H, D, C\}$, is represented by states $(i, j), i \in\left\{t x, t x^{\prime}\right\}$ and $j \in\left[0, T_{n}-1\right]$, with a modeling granularity of aSlotTime $=13 \mu$ s for IEEE $802.11 \mathrm{p}$, and a subframe of $1 \mathrm{~ms}$ for C-V2X Mode 4. Not having a transmission opportunity upon generation requires the packet to wait for an opportunity, which is represented by states $\left(t x^{\prime}, j\right)$, $j \in\left[1, T_{n}-1\right]$. A state transition from $\left(t x^{\prime}, j\right)$ to $(t x, j-1)$, $j \in\left[1, T_{n}-1\right]$, represents a successful transmission. For each technology, we use $\mathcal{P}_{n}^{k}$, where $n \in\{H, D, C\}$ and $k \in\{11 p, v 2 x\}$, to capture the effect of the respective packet

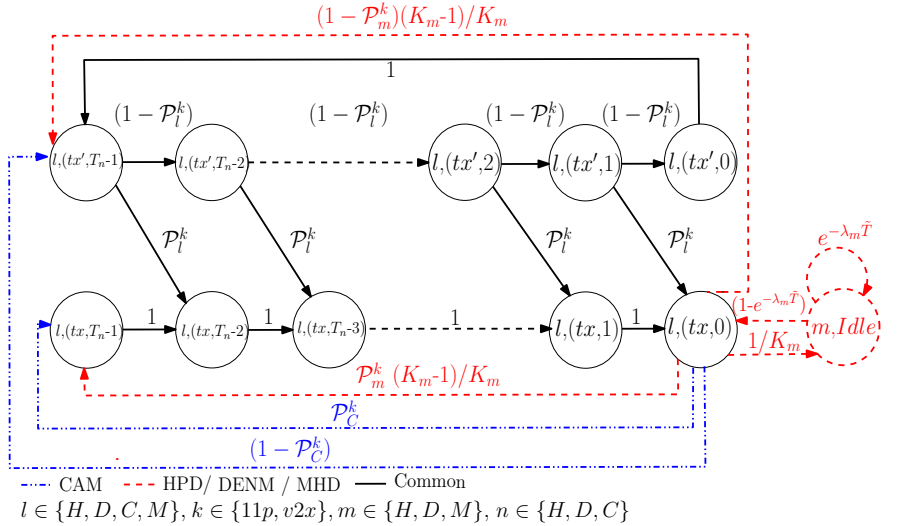

Figure 2: HPD, DENM, CAM and MHD packet generators.

transmitting probabilities in the generator models. To this end, for $n \in\{H, D, C\}, \mathcal{P}_{n}^{11 p}=P_{t, n}^{11 p}$, and $\mathcal{P}_{n}^{v 2 x}=P_{t}^{v 2 x}$, as shown in Figs. 1a and 1b. These parameters link the generators with the DTMCs modeling the MAC layer operation. The variables associated with the generator models are tabulated in Table I at the end of this section for the ease of reference.

\section{B. Packet Queues}

Each packet type has its own queue with the common general structure similar to the one introduced in [14], shown in Fig. 3 [14]. State $(i, l), i \in\left[0, Q^{\max }\right]$ and $l \in\{H, D, C, M\}$, represents a queue of length $i$, such that $Q^{\max }$ is the maximum length. A transition from state $(i, l)$ to state $(i+1, l)$, which is incrementing the queue, or state $(i-1, l)$, which is decrementing the queue, depends on packet generation and transmission, respectively. For $l \in\{H, D, C, M\}$ and $k \in\{11 p, v 2 x\}, P_{q e, l}^{k}$ denotes the probability of having an empty queue, $P_{q n e, l}^{k}=1-P_{q e, l}^{k}$, and $P_{a r r, l}^{k}$ denotes the conditional probability of a new packet of type $l$ arriving given its respective queue is empty. Note that although having the same structure, the transition probabilities of the queue models differ according to the packet type and the technology.

The overall device-level packet queues for the two technologies are different from each other as stated earlier, and they are significantly different to the one presented in [14]. Since [14] does not consider any priority management among the packets, the generated packets are stored in a composite queue in the order of generation, for transmission. The priority handling in this paper is incorporated through the ACs in IEEE $802.11 \mathrm{p}$, thus the queue models can be directly coupled with the appropriate ACs as shown in Fig. 1a. On the other hand, there is only one DTMC to model the MAC layer operations in C-V2X Mode 4. Thus, the four packet-level queues are incorporated such that a resultant queue which simultaneously considers priority levels, is coupled with the DTMC modeling the MAC layer operations, as shown in Fig. 1b. The resultant queue also takes the same structure shown in Fig. 3, and the priority is incorporated through appropriately setting the transition probabilities, which we discuss in detail in Section IV. Similar to the individual queues, $P_{q e}^{v 2 x}$ and $P_{a r r}^{v 2 x}$ denote the queue empty and conditional packet arrival probabilities of the resultant queue, respectively, and $P_{q n e}^{v 2 x}=1-P_{q e}^{v 2 x}$. The variables associated with the queue models are tabulated in Table I at the end of this section for the ease of reference. 


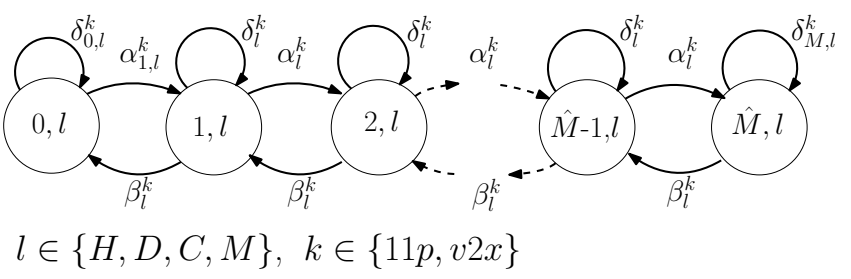

Figure 3: The common queue structure for HPD, DENM, CAM and MHD packets.

\section{DTMC Models for the Four ACs of IEEE 802.11p}

In this subsection, we present the DTMCs used to model the behavior of each AC in IEEE 802.11p. We use index $i \in \mathcal{A C}=$ $\{v o, v i, b e, b k\}$ to differentiate between the parameters related to voice (HPD), video (DENM), best-effort (CAM), and background (MHD), respectively, and the DTMC model illustrated in Fig. 4 is used to describe the MAC layer operations of $A C_{i}$. To this end, vehicle $v$ is at state $\left(I d l e_{i}\right)$, and remains in this state if the packet queue for $A C_{i}$ is empty ${ }^{1}$. When a packet arrives, vehicle $v$ listens for an $A I F S$ duration. The $A I F S$ duration for $A C_{i}$, denoted by $A I F S_{i}$, is calculated according to $A I F S_{i}=a S I F S T i m e+A I F S N_{i} \times$ aSlotTime, where aSlotTime is $13 \mu \mathrm{s}$, aSIFSTime is $32 \mu \mathrm{s}$, and arbitration inter-frame spaces number of $A C_{i}\left(A I F S N_{i}\right)$ is selected according to the AC from the standard [21]. For $A C_{i}$, let $C_{i}$ denote the minimum contention window size, and $\Omega_{i}=A I F S_{i} /$ aSlotTime. States $\left(A_{i}^{j}\right)$ for $j \in\left\{1, \ldots, \Omega_{i}\right\}$ represent this listening time. If the channel stays idle in this duration, the vehicle transmits, which is represented by states $\left(T_{i}, j\right), j \in\{1, \ldots, \vartheta\}$, in which $\vartheta \times$ aSlotTime is the time taken for the transmission. This is in fact the time taken to transmit 134 bytes over a $6 \mathrm{Mbps}$ control channel $(\mathrm{CCH})$ [1]

However, if the channel is found to be occupied within the listening period, the vehicle waits for the transmission to end. This $\vartheta \times$ aSlotTime duration is modeled by states $\left(B_{i}, j\right), j \in\{1, \ldots, \vartheta\}$. State $\left(A_{i}^{1}\right)$ depicts the special case of a packet arrival while the channel is busy. In such a case, the vehicle may not have to wait for $\vartheta \times a$ SlotTime until the channel becomes free again. Thus, the waiting duration can be written as $J \times a$ SlotTime, where integer $J$ is random and uniform in $[1, \vartheta]$. Therefore, the transition from state $\left(A_{i}^{1}\right)$ is different from the transitions from $\left(A_{i}^{j}\right)$ for $j \in\left\{2, \ldots, \Omega_{i}\right\}$. We can also notice a difference in the transition probabilities. The two transition probabilities represent slight variations of the probability of the channel being busy. At states $\left(A_{i}^{j}\right)$, where $j \in\left\{2, \ldots, \Omega_{i}\right\}$, the channel has remained idle for at least one aSlotTime interval. Thus, if vehicle $v$ can sense transmission at one of these states, the transmission from a neighboring vehicle must have just initiated. Hence, the transmitting vehicle should be at the first transmitting state. This restriction does not exist at $\left(A_{i}^{1}\right)$, and the transmitting vehicle can be at any transmitting state.

After vehicle $v$ reaches $\left(B_{i}, \vartheta\right)$, where the channel is supposed to be idle again, it initiates a backoff by randomly and uniformly selecting a backoff counter value from $\left[0, C_{i}\right]$. Each counter value gives rise to a specific backoff stage with both counter values zero and one leading to stage zero,

\footnotetext{
${ }^{1}$ This paper presents a more precise definition of state $\left(I_{d l e_{i}}\right)$ compared to the one in [25]. The subsequent derivations and the expressions for performance indicators differ accordingly.
}

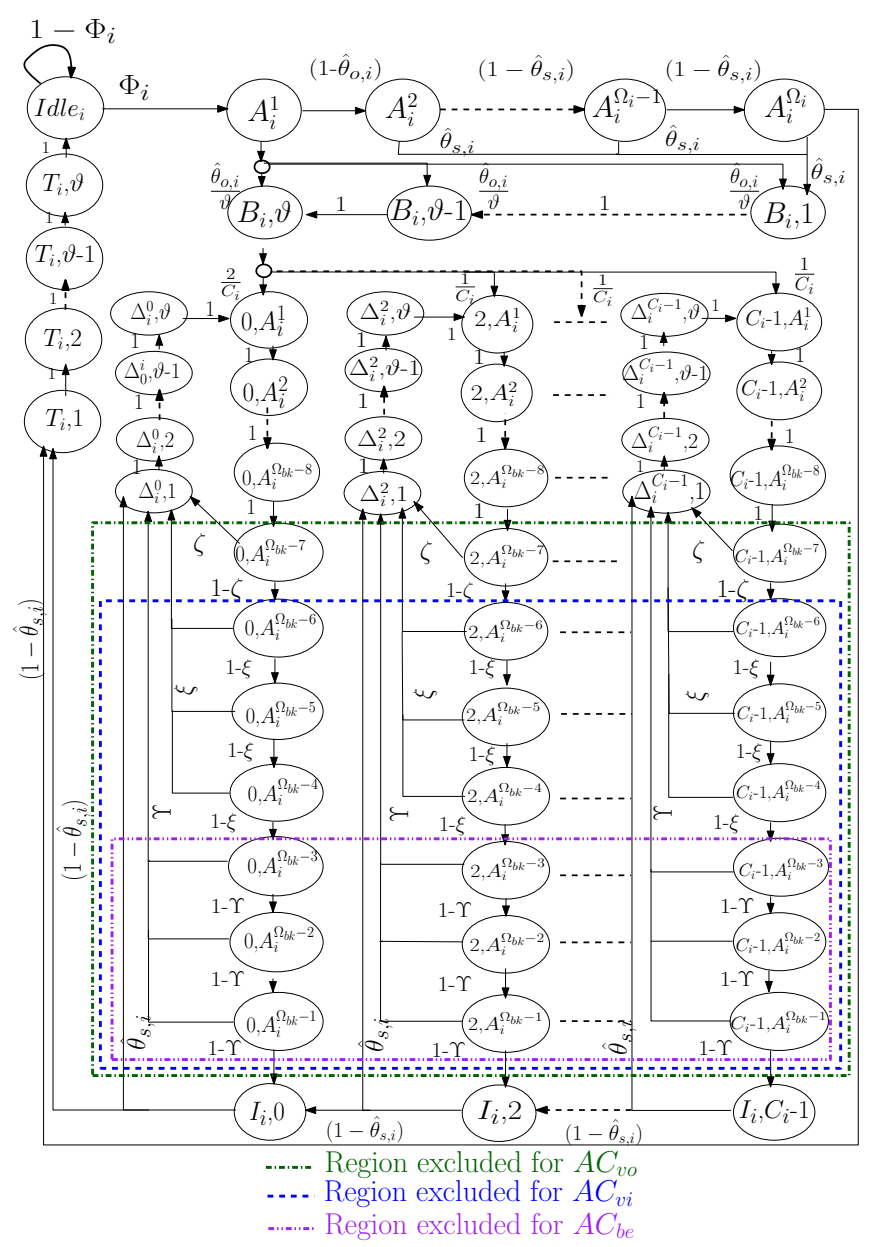

Figure 4: DTMC models for the MAC layer operations of the four ACs in IEEE 802.11p. according to the standard. During backoff, the vehicle waits for an $A I F S$ duration before sensing the channel again, and this wait is represented by states $\left(b, A_{i}^{j}\right), j \in\left\{1, \ldots,\left(\Omega_{i}-1\right)\right\}$, for counter value $b \in\left\{0, \ldots,\left(C_{i}-1\right)\right\}$. During the wait, the vehicle senses the channel after each aSlotTime interval. If it finds the channel to be busy, it waits $\vartheta \times a$ SlotTime for the transmission of the neighboring vehicle to end. This duration is represented by states $\left(\Delta_{i}^{b}, j\right)$, for $j \in\{1, \ldots, \vartheta\}$. Upon the completion of the transmission of the neighboring vehicle, the vehicle waits for another $A I F S$ duration at the same backoff stage. The process continues until the channel is found to be free at state $\left(I_{i}, b\right)$. Observation of a free channel at state $\left(I_{i}, b\right)$ leads to decrementing the backoff counter to arrive at state $\left(I_{i}, b-1\right)$. The channel being free at state $\left(I_{i}, 0\right)$ gives the vehicle the opportunity to transmit.

The ACs with a higher priority have a shorter $A I F S$ duration, and $A C_{b k}$, which is the $\mathrm{AC}$ with the lowest priority, has the longest duration. Thus, Fig. 4 in fact illustrates the DTMC model for $A C_{b k}$. The DTMC models for the other three ACs that have shorter $A I F S$ duration values can be obtained by appropriately excluding states and transitions (as illustrated) from Fig. 4. For the backoff stage value $b$, the transition from states $\left(b, A_{i}^{\Omega_{b k}-j}\right)$, where $j \in\left\{1, \ldots,\left(\Omega_{b k}-\Omega_{v o}\right)\right\}$, to state $\left(\Delta_{i}^{b}, 1\right)$, can only happen due the arrival of a higher priority packet. As an example, for $A C_{b k}$, this can happen due to the arrival of a HPD, DENM or CAM packet. Let the channel busy ratio $\theta_{i}$ denote the probability of the channel being busy for 
$A C_{i}$. Then, $\zeta=\theta_{v o}, \xi=\left(\theta_{v o}+\theta_{v i}\right)$, and $\Upsilon=\sum_{i \in \mathcal{A C} \backslash\{b k\}} \theta_{i}$. The variables associated with the DTMC are tabulated in Table I at the end of this section for the ease of reference.

\section{DTMC Model for C-V2X Mode 4}

C-V2X Mode 4 utilizes the SPS algorithm for radio resource allocation [8]. The SPS algorithm is specially designed for identifying the available candidate single-subframe resources (CSRs) and allocating them for communication without the aid of the cellular infrastructure. Upon allocation, the vehicle uses the radio resource $R C$ times, where $R C$ is called the reselection counter. The value $R C$ is selected uniformly and randomly from $\left[R_{l}, R_{h}\right]$, where $R_{l}$ and $R_{h}$ denote the lower and upper bounds for $R C$, respectively. Let $P_{s c h}$ denote the probability of allocating a suitable CSR for a vehicle by utilizing the SPS algorithm. $\Gamma$ denotes the length of the selection window. This is the time allocated for CSR selection, and is defined as the maximum tolerable latency in the standard [7]. Each selection window size has its respective range for $R C$. According to the standard, the three selection window sizes are, $\Gamma=100 \mathrm{~ms}$ with $R C \in[5,15], \Gamma=50 \mathrm{~ms}$ with $R C \in[10,30]$ and $\Gamma=20 \mathrm{~ms}$ with $R C \in[25,75]$ [6].

We assume that $P_{s c h}=1$ without any loss of generality as it is unlikely that a vehicle ends up without any radio resources to transmit according to the standard. However, the selection window size should be appropriately increased with the number of vehicles in the network to maintain this condition. Let $C S R_{t o t, \Gamma}$ denote the total number of CSRs in a selection window of $\Gamma$ duration. For example, according to [7], a 1 ms subframe contains 100 resource blocks (RB). Transmitting a payload of 100 bytes using 64 QAM modulation requires at least 4 RBs. Thus, a subframe can hold 25 CSRs, which means $C S R_{t o t, 20}=500$. The maximum number of vehicles that the system can support for each selection window size while maintaining $P_{s c h}=1$ is denoted by $N_{m, \Gamma}=0.8 \times C S R_{t o t, \Gamma}$ according to the standard [8]. The DTMC model for the MAC layer operations is illustrated in Fig. 5. We note that this model for C-V2X Mode 4 was first introduced in [14]. We present a summary of this model for completeness.

State Idle represents the idle state with either no packets or no CSRs to transmit. The waiting time before transmitting a newly arrived packet is modeled using states $(w, j)$, $j \in[0, \Gamma-2]$, and these states are assumed equiprobable. The vehicle uniformly selects a random value for $R C$ after this waiting period. States $(i, 0)$, where $i \in\left[1, R_{h}\right]$ depicts the current $R C$ value, represent transmission opportunities. Upon a transmission opportunity, the system stays in the current $R C$ value for $\Gamma \mathrm{ms}$ if the queue is empty, or otherwise, transmits and moves to $(R C-1, \Gamma-1)$. If the queue is not empty at $(1,0)$, the vehicle opts for a new radio resource with probability $1-P_{r k}$, or else continue using the same radio resource, where $P_{r k} \in[0,0.8]$ according to the standard [8]. The variables associated with all the DTMCs presented in this section are tabulated in Table I for the ease of reference.

\section{Evaluation of the Dependencies AMONG THE DTMCS}

In this section, we derive expressions for the parameters that introduce dependencies among the DTMCs as shown in
Table I: The parameters of the analytical models.

\begin{tabular}{|c|c|}
\hline Parameters & Description \\
\hline \multicolumn{2}{|c|}{$\begin{array}{l}\text { For } i \in\{v o, v i, b e, b k\}, m \in\{H, D, M\}, l \in\{H, D, C, M\} \\
n \in\{H, D, C\}, \text { and } k \in\{11 p, v 2 x\}: \\
\text { Generator models }\end{array}$} \\
\hline$\lambda_{m}$ & Arrival rate of packet $m$ \\
\hline $\mathcal{P}_{l}^{k}$ & The transmission probability of packet $l$. \\
\hline$K_{m}$ & Number of repetitions of packet $m$. \\
\hline$T_{n}$ & Repetition interval of packet $n$. \\
\hline \multicolumn{2}{|c|}{ Queue models } \\
\hline$Q^{\max }$ & The maximum queue length. \\
\hline$P_{q e, l}^{k}$ & Queue empty probability of packet $l$. \\
\hline $\begin{array}{l}q e, x \\
P_{q e}^{v 2 x} \\
P^{k}\end{array}$ & $\begin{array}{l}\text { Queue empty probability of the C-V2X resultant queue. } \\
1-P^{k}\end{array}$ \\
\hline $\begin{array}{l}q n e, l \\
P_{a r r}^{v 2 x}\end{array}$ & $\begin{array}{l}\text { Conditional packet arrival probability of the } \mathrm{C}-\mathrm{V} 2 \mathrm{X} \\
\text { resultant queue. }\end{array}$ \\
\hline \multicolumn{2}{|c|}{ IEEE 802.11p model } \\
\hline$A I F S N_{i}$ & Arbitration inter-frame spacing number of $A C_{i}$. \\
\hline$A I F S_{i}$ & Arbitration inter-frame spacing of $A C_{i}$ \\
\hline $\begin{array}{l}\text { aSlotTime } \\
\Omega_{i}\end{array}$ & $\begin{array}{l}\text { The smallest time unit of the IEEE } 802.11 \mathrm{p} \text { protocol }(13 \mu \mathrm{s}) \\
\text { AIF } S_{i} / \text { aSlotTime. }\end{array}$ \\
\hline i & $\begin{array}{l}\text { The number of aSlotTime taken to transmit a } 134 \text { bytes } \\
\text { packet over a } 6 \mathrm{Mbps} \mathrm{CCH} \text {. }\end{array}$ \\
\hline & The minimum contention window size for $A C_{i}$ \\
\hline \multicolumn{2}{|c|}{ C-V2X Mode 4 model } \\
\hline$\Gamma$ & The selection window size. \\
\hline$P_{r k}$ & The probability of using the same radio resource. \\
\hline$P_{\text {sch }}$ & $\begin{array}{l}\text { The probability of allocating a suitable CSR for a vehicle } \\
\text { by utilizing the SPS algorithm. }\end{array}$ \\
\hline$R_{h}$ & The upper bound for $R C$. \\
\hline$R_{l}$ & The lower bound for $R C$. \\
\hline$R C$ & The reselection counter value. \\
\hline
\end{tabular}

Figs. 1a and 1b, by utilizing the steady-state solutions of each DTMC. We denote the steady-state probability of an arbitrary state $(A)$ by $\pi_{A}^{k}$, where $k \in\{11 p, v 2 x\}$. We first focus on the generator models.

\section{A. Generator Models}

The parameters that introduce dependencies among the generators and the queue models are the queue advancing transition probabilities $\alpha_{l}^{k}$ and $\alpha_{1, l}^{k}$, and the queue servicing transition probabilities $\beta_{l}^{k}$, for $l \in\{H, D, C, M\}$ and $k \in\{11 p, v 2 x\}$. To this end, we have $\alpha_{i}^{k}=\pi_{i,\left(t x^{\prime}, 0\right)}^{k}, \alpha_{1, i}^{k}=$ $\pi_{i,(t x, 0)}^{k}\left(1-\frac{1}{K_{i}}\right)\left(1-\mathcal{P}_{i}^{k}\right), \quad$ for $i \in\{H, D\}, \alpha_{C}^{k}=$ $\pi_{C,\left(t x^{\prime}, 0\right)}^{k}, \alpha_{1, C}^{k}=\pi_{C,(t x, 0)}^{k}\left(1-\mathcal{P}_{C}^{k}\right)$, and $\alpha_{1, M}^{k}=\alpha_{M}^{k}=$ $\left(1-e^{-\lambda_{M} \tilde{T}}\right)$, where $k \in\{11 p, v 2 x\}$. Moreover, $\mathcal{P}_{n}^{11 p}=P_{t, n}^{11 p}$ and $\mathcal{P}_{n}^{v 2 x}=P_{t}^{v 2 x}$ for $n \in\{H, D, C\}$, and the expressions for the steady-state probabilities of the generator models are presented in Appendix A.

The queue servicing transition probabilities related to the four queue models associated with IEEE $802.11 \mathrm{p}$ are given by $\beta_{n}^{11 p}=P_{t, n}^{11 p} \sum_{j=1}^{T_{n}-1} \pi_{n,\left(t x^{\prime}, j\right)}^{11 p}$, for $n \in$ $\{H, D, C\}$, and $\beta_{M}^{11 p}=P_{t, M}^{11 p}$, where $P_{t, M}^{11 p}$ denotes the transmit probability of MHD packet in IEEE 802.11p. Although the queue advancing transition probabilities are of similar form for both enabling technologies, there is a variation in the queue servicing transition probabilities since the priority management is incorporated in the queue models for C-V2X Mode 4. This means, the packets in the lower priority queues are only served if there are no packets in the higher priority queues, thus we have $\beta_{H}^{v 2 x}=$ $\sum_{j=1}^{T_{H}-1} \pi_{H,\left(t x^{\prime}, j\right)}^{v 2 x} P_{t}^{v 2 x}, \beta_{D}^{v 2 x}=\sum_{j=1}^{T_{D}-1} \pi_{D,\left(t x^{\prime}, j\right)}^{v 22} P_{t}^{v 2 x} P_{q e, H}^{v 2 x}$, 


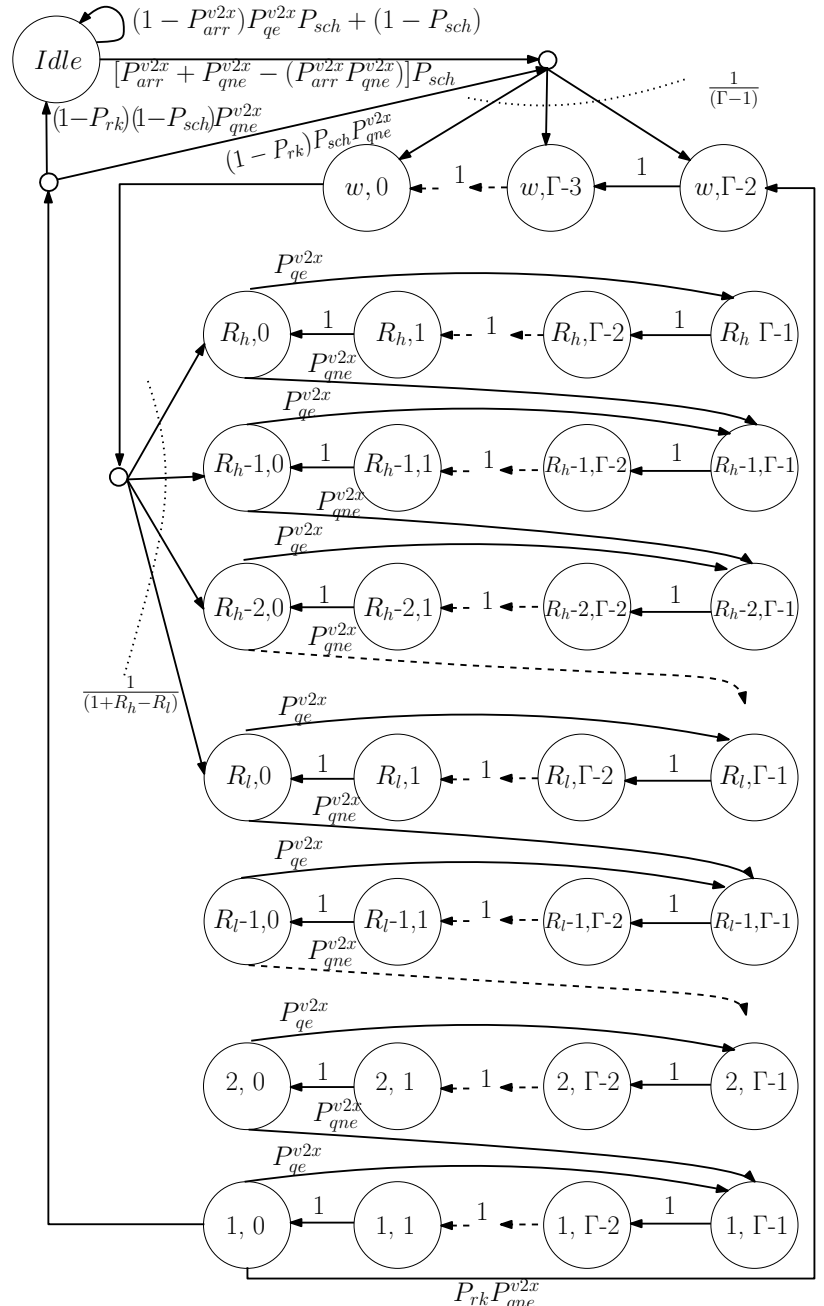

Figure 5: DTMC model for the MAC layer operations of C-V2X Mode 4. $\beta_{C}^{v 2 x}=\sum_{j=1}^{T_{C}-1} \pi_{C,\left(t x^{\prime}, j\right)}^{v 2 x} P_{t}^{v 2 x} P_{q e, H}^{v 2 x} P_{q e, D}^{v 2 x}$, and $\beta_{M}^{v 2 x}=$ $P_{t}^{v 2 x} \prod_{n \in\{H, D, C\}} P_{q e, n}^{v 2 x}$.

We can also observe dependencies between the generator models and the DTMCs introduced for the MAC layer operations. To this end, for $k \in\{11 p, v 2 x\}$, we have $P_{a r r, m}^{k}=$ $\left(1-e^{-\lambda_{m} \tilde{T}}\right)$ for $m \in\{H, D, M\}$, and $P_{a r r, C}^{k}=\pi_{t x, 0}^{C}+\pi_{t x^{\prime}, 0}^{C}$. The packet arrival probability for the resultant queue in $\mathrm{C}$ V2X Mode 4 is given by $P_{a r r}^{v 2 x}=P_{a r r, H}^{v 2 x}+P_{a r r, D}^{v 2 x} P_{q e, H}^{v 2 x}+$ $P_{a r r, C}^{v 2 x} P_{q e, H}^{v 2 x} P_{q e, D}^{v 2 x}+P_{a r r, M}^{v 2 x} \prod_{n \in\{H, D, C\}} P_{q e, n}^{v 2 x}$.

\section{B. Quеиe Models}

Next, we focus on the dependencies among the queue models and the DTMCs for the MAC layer operations, which are the probabilities of the queues being empty. These values can be obtained through the steady-state probability of the zeroth state in each queue, and can be written as $\pi_{0, l}^{k}=\left[1+\alpha_{1, l}^{k}\left(\frac{1-\left(\beta_{l}^{k}\right)^{-Q^{\max }}\left(\alpha_{l}^{k}\right)^{Q^{\max }}}{\beta_{l}^{k}-\alpha_{l}^{k}}\right)\right]^{-1}=P_{q e, l}^{k}$, for $l \in\{H, D, C, M\}$ and $k \in\{11 p, v 2 x\}$. The queue empty probability of the resultant queue in C-V2X Mode 4 is given by $P_{q e}^{v 2 x}=\prod_{l \in\{H, D, C, M\}} P_{q e, l}^{v 2 x}$.

\section{Models for the MAC Layer Operations}

The transmission probabilities introduce the dependencies between the DTMCs modeling the MAC layer operations and their associated generator models. Further evaluation of these parameters requires the steady-state solutions and the transition probabilities of the DTMCs modeling the MAC layer operations. The steady-state solutions of the DTMCs modeling the different ACs in IEEE 802.11p are presented in Appendix A. With regards to the transition probabilities in Fig. 4, it is not hard to show that $\Phi_{v o}=[1-(1-$ $\left.\left.P_{a r r, H}^{11 p}\right) P_{q e, H}^{11 p}\right], \Phi_{v i}=\left[1-\left(1-P_{a r r, D}^{11 p}\right) P_{q e, D}^{11 p}\right], \Phi_{b e}=[1-$ $\left.\left(1-P_{a r r, C}^{11 p}\right) P_{q e, C}^{11 p}\right]$, and $\Phi_{b k}=\left[1-\left(1-P_{a r r, M}^{11 p}\right) P_{q e, M}^{11 p}\right]$. These values can be computed utilizing the dependency parameters from the queue and generator models. Moreover, we consider $\hat{\theta}_{s, i} \approx 1-\left[\prod_{i \in \mathcal{A C}}\left(1-\pi_{T_{i}, 1}\right)\right]^{N-1}+\sum_{k \in \mathcal{A C} \backslash\{i\}}\left(\pi_{T_{k}, 1}\right)$ and $\hat{\theta}_{o, i} \approx 1-\left[\prod_{i \in \mathcal{A C}}\left(1-\sum_{j=1}^{\vartheta} \pi_{T_{i}, j}\right)\right]^{N-1}+$ $\left[\sum_{k \in \mathcal{A C} \backslash\{i\}} \sum_{j=1}^{\vartheta}\left(\pi_{T_{k}, j}\right)\right]$, which can be used subsequently to obtain expressions for $\theta_{i}$ for $i \in \mathcal{A C}$. For an example, $\theta_{v o}=$ $\left(\sum_{i \in \mathcal{A C}} \hat{\theta}_{s, i}\right)\left(\pi_{A_{v o}^{\Omega_{v o}}}+\pi_{I_{v o}, 0}\right) /\left[4 \sum_{i \in \mathcal{A C}}\left(\pi_{A_{i}^{\Omega_{i}}}+\pi_{I_{i}, 0}\right)\right]$. With these transition probabilities, the transmit probability of each packet type can be obtained by summing the steady-state probabilities of the transmit states of the appropriate $\mathrm{AC}$, i.e., $P_{t, l}^{11 p}=\sum_{j=1}^{\vartheta} \pi_{T_{i}, j}$ for $l \in\{H, D, C, M\}$, where index $i$ represents the appropriate $\mathrm{AC}$ such that $i \in \mathcal{A C}$.

A similar approach can be used to obtain an expression for $P_{t}^{v 2 x}$. Firstly, the transition probabilities can be calculated utilizing the dependency parameters from the queue and generator models. Then, the probability of transmission opportunity can be found by summing the steady state probabilities of the transmit states, i.e., $P_{t x o}=\sum_{j=1}^{R_{h}} \pi_{j, 0}$, where the expressions for the steady-state probabilities are listed in Appendix A. Finally, we have $P_{t}^{v 2 x}=P_{t x o} \times P_{q n e}^{v 2 x}$.

\section{PERformance AnAlysis}

This section focuses on deriving expressions for few key performance metrics at the MAC layer.

\section{A. Average Delay}

Firstly, we focus on the average delay between generation and transmission. The delay value captures the waiting time in the queue (queuing delay), and the delay in accessing radio resources (access delay). This can be calculated through the cycle time of the DTMCs modeling the MAC layer operations, and the steady-state probabilities of the DTMCs modeling the queues. We present expressions for the average delay through the following lemma.

Lemma 1: Let index $i \in \mathcal{A C}$ represent the respective $\mathrm{AC}$ for $l \in\{H, D, C, M\}$. The average delay for multi-priority data streams in IEEE $802.11 \mathrm{p}$ is given by $d_{a v g, l}^{11 p}=\operatorname{aSlotTime}[(1-$ $\left.\left.\pi_{i d l e_{i}}\right) / \pi_{T_{i}, 1}+\vartheta-1\right] \sum_{j=0}^{Q^{\max }}(j+1) \pi_{j, l}^{11 p}$. For C-V2X Mode 4 , it is given by $d_{a v g, l}^{v 2 x}=\sum_{j=1}^{Q^{\max }} \frac{2 j-1}{2 P_{t x o}} \pi_{j, l}^{v 2 x} /\left(1-P_{q e, l}^{v 2 x}\right)$.

Proof: See Appendix B.

\section{B. Probability of Collision}

According to the IEEE 802.11p standard, a collision occurs when two or more vehicles initiate their transmission simultaneously, or two or more data streams of a given vehicle initiate the transmission simultaneously (self-collision). On the other 
hand, a collision occurs in C-V2X Mode 4 due to an overlap in the selection windows of nearby vehicles, as explained in [14]. The overlap may lead to them choosing the same CSR for transmission. The expressions for collision probability are as follows.

Lemma 2: The collision probability of IEEE $802.11 \mathrm{p}$ and CV2X Mode 4 are given by $P_{c o l}^{11 p}=2-\left[\prod_{i \in \mathcal{A C}}\left(1-\pi_{T_{i}, 1}\right)\right]^{N}-$ $N \sum_{i \in \mathcal{A C}}\left(\pi_{T_{i}, 1} \theta_{i}\right)\left[\prod_{j \in \mathcal{A C}}\left(1-\pi_{\left.T_{j}, 1\right)}\right]^{N-1}-\left[\prod_{i \in \mathcal{A C}}(1-\right.\right.$ $\left.\left.\pi_{T_{i}, 1}\right)\right]-\sum_{i \in \mathcal{A C}} \pi_{T_{i}, 1} \prod_{j \in \mathcal{A C} \backslash\{i\}}\left(1-\pi_{T_{j}, 1}\right)$ and $P_{c o l}^{v 2 x} \approx$ $1-\left[1-\left[1-\prod_{j=0}^{\Gamma-1}\left(1-\frac{\pi_{1,0}}{1-j \pi_{1,0}}\right)\right] \frac{\left(1-P_{r k}\right)}{\left(C S R_{t o t, \Gamma}-N+1\right)}\right]^{N-1}$, respectively.

Proof: See Appendix B.

\section{Average Channel Utilization and Throughput}

The average channel utilization is the fraction of time any available channel resource is used for transmission by at least one vehicle. The channel resource is the shared channel in IEEE 802.11p and any CSR in C-V2X Mode 4. In IEEE $802.11 p$, the channel utilization captures the probability of at least one vehicle transmitting, thus it can be written as $C U_{a v g}^{11 p}=1-\left[\prod_{i \in \mathcal{A C}}\left(1-\sum_{j=1}^{\vartheta} \pi_{T_{i}, j}\right)\right]^{N}$. Moreover, the total throughput is the product of the bandwidth of the $\mathrm{CCH}$ and the probability of exactly one vehicle transmitting, which is given by $S^{11 p}=$ Bandwidth of $\mathrm{CCH} \times$ $N \sum_{i \in \mathcal{A C}}\left(\sum_{j=1}^{\vartheta} \pi_{T_{i}, j} \theta_{i}\right)\left[\prod_{i \in \mathcal{A C}}\left(1-\sum_{j=1}^{\vartheta} \pi_{T_{i}, j}\right)\right]^{(N-1)}$.

For C-V2X Mode 4, we are interested in finding the average channel utilization within a single subframe. Therefore, channel utilization is normalized by $C S R_{t o t, \Gamma} / \Gamma$, which is the total number of CSRs per subframe. Thus, the average channel utilization can be written as $C U_{a v g}^{v 2 x}=P_{t}^{v 2 x} N(1-$ $\left.P_{c o l}^{v 2 x}\right) /[$ CSRs per Subframe]. Moreover, the throughput can be written as $S^{v 2 x}=$ Channel Bandwidth $\times C U_{a v g}^{v 2 x}$.

\section{NUMERICAL RESULTS AND DISCUSSION}

In this section, we present numerical results to compare the MAC layer performance of IEEE 802.11p and C-V2X Mode 4 in the presence of concurrent multi-priority data streams. The reference packet formats of HPD, DENM, CAM, and MHD are set according to [2], [3]. We set $T_{H}=100 \mathrm{~ms}$ and $K_{H}=8$ for HPD, and $T_{D}=500 \mathrm{~ms}$ and $K_{D}=5$ for DENM, to capture the difference in priority levels [3]. The standard in fact allows the vehicle to choose $T_{i}$ and $K_{i}, i \in\{H, D\}$, based on the severity of the event. Moreover, we consider $T_{C}$ to be between $100 \mathrm{~ms}$ and $1 \mathrm{~s}$ [2], and we set $K_{M}=1$. Adjusting $T_{C}$ dynamically according to network performance is termed as adaptive CAM rate. The value of $\lambda_{m}$, for $m \in\{H, D, M\}$, is set at 0.1 packets/s, by considering the example use case scenarios in [26]. For the purpose of maintaining $P_{s c h}=1$, we have $N_{m, 20}=400, N_{m, 50}=1000$ and $N_{m, 100}=2000$. We set $P_{r k}=0.4$. In IEEE $802.11 \mathrm{p}$, the $\mathrm{CCH}$ has a bandwidth of $10 \mathrm{MHz}$, and the transmission data rate is $6 \mathrm{Mbps}$ [27]. C-V2X Mode 4 utilizes a channel bandwidth of $10 \mathrm{MHz}$ with a transmission data rate of $20 \mathrm{Mbps}$ [17].

Steady-state probabilities of the DTMCs are computed iteratively according to the flowcharts in Figs. 1a and 1b.
For example, in IEEE 802.11p, we start solving the generator models sequentially according to their priority and then solve the queue models sequentially according to priority. Finally, we sequentially solve the AC models according to their precedence. This process is continued iteratively until the parameters converge. The rate of convergence was not found to be a bottleneck in the numerical evaluations, and all parameters in the models did exhibit fast convergence. For example, the values for $P_{q e, M}^{11 p}$ and $P_{q e, M}^{v 2 x}$ converge after approximately 25 and 5 iterations, respectively, at $N=50$. The convergence rate increases with $N$ for IEEE $802.11 \mathrm{p}$ and reduces slightly in $\mathrm{C}-\mathrm{V} 2 \mathrm{X}$ Mode 4 with increasing $N$. It was also observed that the parameters of the C-V2X Mode 4 models converge faster than its counterpart.

The parameter values are used subsequently to calculate the performance measures introduced in Section IV, and we first compare the average delay. We validate the results obtained for IEEE 802.11p and C-V2X Mode 4 using simulation tools NS3 and Matlab, respectively. For the IEEE 802.11p model validation, we have used the NS3 code in [28], and we have modified it to support multi-priority packets and multiple queues. Then, we have obtained the data for validation through the simulation traces. For C-V2X Mode 4, we have first implemented the periodic and Poisson arrivals for packet generation. Then, we have used four arrays to model the queues of the generated packet types. The SPS algorithm is then implemented according to the standard [8], and the results are generated. Since C-V2X Mode 4 stems on a scheduling based access mechanism, a discrete-event simulator is not mandatory for the validation, and hence, we have resorted to a much simpler validation process using Matlab. The validation results are presented by considering the parallel operation of all four data streams. Please note that validation results for some curves have not been included as they affect the clarity of the figures.

\section{A. Average delay}

The average delay is calculated according to Lemma 1, and the results are illustrated in Fig. 6. As discussed in [14], it is clear that IEEE 802.11p displays a lower average delay compared to $\mathrm{C}-\mathrm{V} 2 \mathrm{X}$ Mode 4, thanks to the relatively short $A I F S$ values when compared with the selection window sizes in C-V2X Mode 4. The figures clearly illustrate priority management, with the higher priority data streams experiencing lower average delays, in both technologies. In IEEE 802.11p, this is achieved by allocating lower $A I F S$ values and smaller contention window sizes for higher priority ACs, whereas in C-V2X Mode 4, this is achieved by giving preference to higher priority packets at every transmission opportunity.

In particular, Fig. 6 illustrates the behavior of the average delay with $N$. We use the term single stream to refer to scenarios in which only one packet type is used for communication, as in [14], [17], [29], and the term multiple streams to refer to scenarios where the parallel operation of all four data streams is considered as per the models in this paper. The plots clearly highlight the importance of the motivation behind this paper, as there is a large deviation, in particular for the lower priority packets. Fig. 6a confirms the effectiveness 


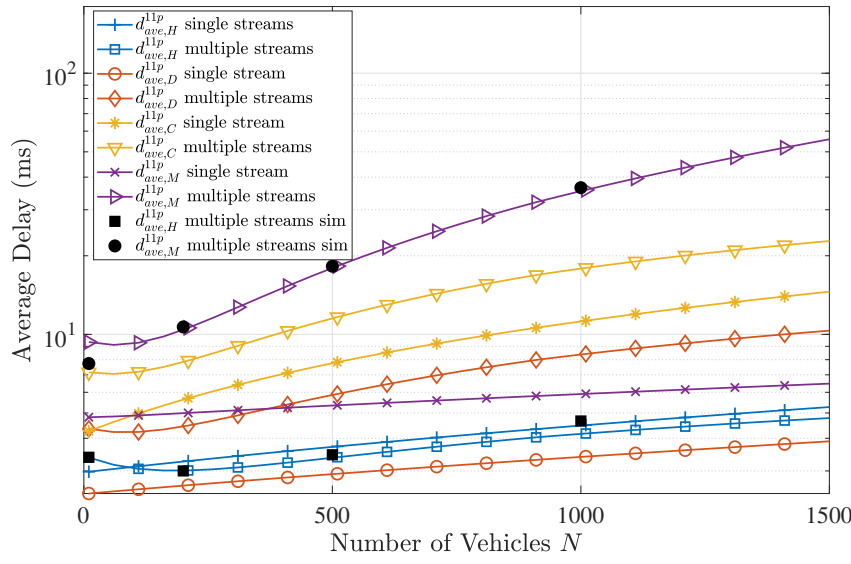

(a) IEEE $802.11 \mathrm{p}$, where $T_{C}=100$.

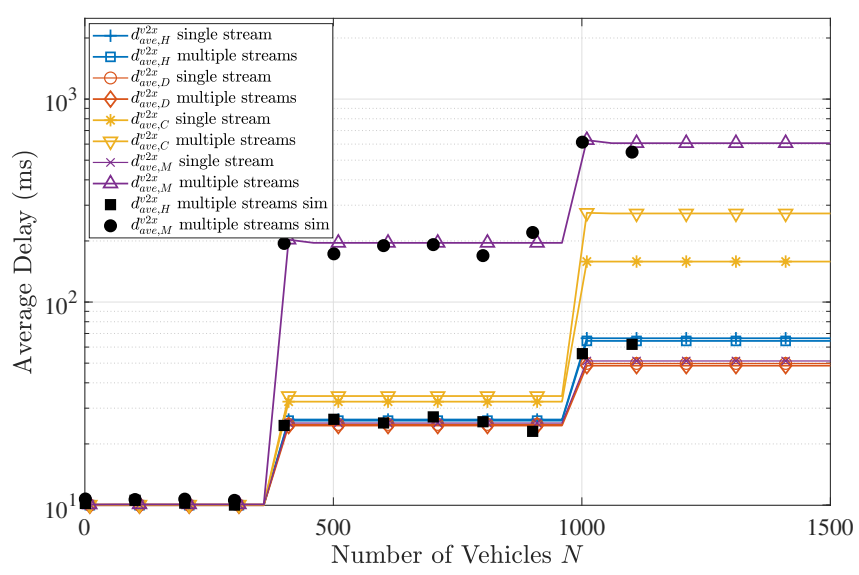

(b) C-V2X Mode 4, where $T_{C}=100$.

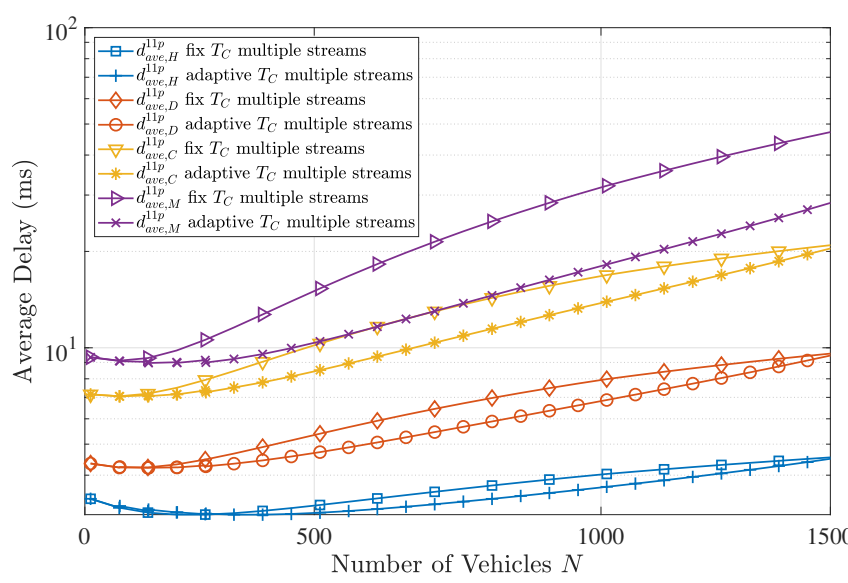

(c) IEEE $802.11 \mathrm{p}$ with adaptive CAM rates.

Figure 6: The behavior of the average delay with $N$.

of the EDCA mechanism in IEEE 802.11p [25]. According to ETSI standard [26], the maximum allowable latency in V2X communication is recommended to be less than $100 \mathrm{~ms}$ for most use cases. We can see that IEEE 802.11p satisfies this requirement on the average for packets of all priority levels, even in ultra-dense network settings. This is not the case with C-V2X Mode 4, as shown in Fig. 6b. The lower priority data streams easily exceed this threshold value as soon as the network becomes dense. However, HPD and DENM packets, which have the most crucial latency constraints, satisfy the $100 \mathrm{~ms}$ delay constraints on the average, even in ultra-dense networks.

Being consistent with [14], it can be noted from Fig. 6b that the average delay in C-V2X Mode 4 is mainly sensitive to $\Gamma$, exhibiting a step-wise increase when $\Gamma$ switches from a shorter to a longer value. Increasing $\Gamma$ reduces $P_{t x o}$, which in turn increases $d_{a v g, l}^{v 2 x}$ in Lemma 1. For the CAM and MHD curves, the average delay for the single stream is lower than multiple streams, which is rather intuitive. However, for HPD and DENM, we can observe the average delay slightly decreasing when switching from single to multiple streams. It has been shown in [14] that selecting new CSR values leads to a reduction in average delay compared to retaining the same CSR value. When considering multiple parallel data streams, there are more packets to transmit, thus the vehicle gets the chance to select new CSR values $(R C=1$ condition) more frequently, which leads to this slight reduction. It is interesting to note that Fig. 6b illustrates insignificant difference in average delay among data streams when $\Gamma=20 \mathrm{~ms}$. Small values of $\Gamma$ lead to frequent transmission opportunities, and negligible queue build up. This is not true when $\Gamma$ is large, thus we can observe a significant variation in the queuing delays among the multi-priority data streams.

Note that the difference in the average delay between the highest (HPD) and the lowest (MHD) priority data streams is higher in C-V2X Mode 4 compared to IEEE 802.11p. Thus, IEEE $802.11 \mathrm{p}$ can be considered to be fairer among multipriority data steams with regards to average delay. The ETSI ITS-G5 decentralized congestion control (DCC) algorithm for IEEE $802.11 \mathrm{p}$ proposes regulating $T_{C}$ according to the utilization of the channel for congestion control [14]. To this end, $T_{C}$ can be increased to reduce the congestion. The reduction in congestion in turn further reduces the delay in all data streams as shown in Fig. 6c. There is no built-in technique to control $T_{C}$ in C-V2X Mode 4 adaptively. This is an interesting avenue that can be further explored in future releases of $\mathrm{C}-\mathrm{V} 2 \mathrm{X}$ Mode 4.

\section{B. Collision probability}

The collision probability is calculated according to Lemma 2, and the results are illustrated in Fig. 7. The collision probability obviously increases with $N$. However, it is interesting to note that C-V2X Mode 4 experiences a lower collision probability than IEEE $802.11 \mathrm{p}$. This implies that the SPS algorithm is superior for collision resolution compared to its contention-based counterpart. Adaptive CAM rate leads to a marginal improvement in the collision probability of IEEE 802.11 p, but it is still comparatively high for $N>300$. The observations imply that C-V2X Mode 4 is the preferable choice for ultra-reliable communications.

The behavior of the collision probability of IEEE $802.11 \mathrm{p}$ with $N$ is illustrated in Fig. 7a. The figure also highlights the significance of the motivation behind this paper, as there is a considerable deviation between the single and parallel multiple streams. The collision probabilities are expected to increase further with increasing packet generation rates. The equivalent results for C-V2X Mode 4 are illustrated in Fig. 7b. We can 


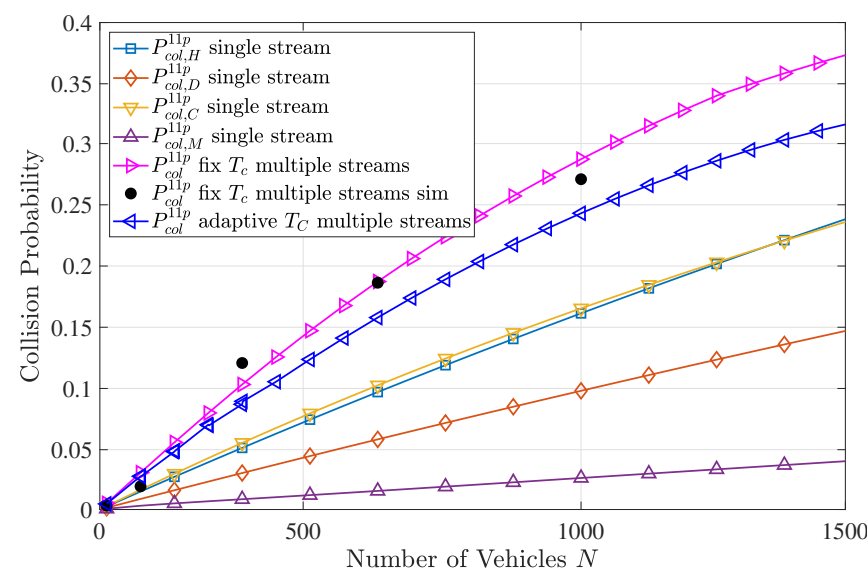

(a) IEEE $802.11 \mathrm{p}$.

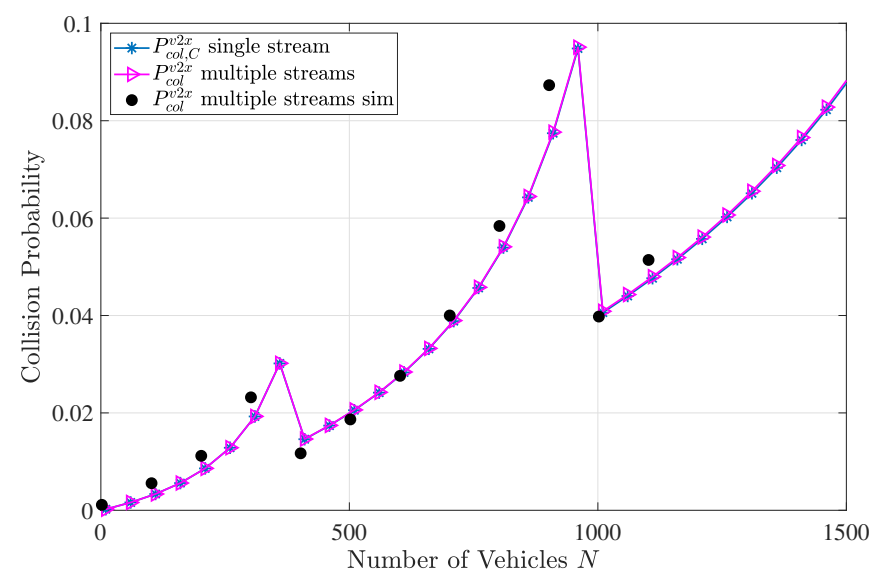

(b) C-V2X Mode 4.

Figure 7: The behavior of the collision probability with $N$, where $T_{C}=100 \mathrm{~ms}$. observe the collision probability increasing exponentially with $N$. When considering the single stream operations, there is no major variation among the collision probabilities of the four packet types. A similar observation can be made when comparing with parallel multiple streams. Thus, only two curves are presented for clarity. The minor variations are due to C-V2X Mode 4 using a schedule-based access mechanism. We note that $\Gamma$ has a significant impact on the collision probability. When $\Gamma$ is increased to preserve $P_{s c h}=1$, i.e., at $N=400$ and $N=1000$, the collision probability reduces significantly, as $C S R_{t o t, \Gamma}$ increases when a vehicle switches from a shorter to a longer selection window. The overall curve can be shifted down further if we reduce the values of $N$ at which the selection window switches, e.g., we switch it at $N=300$ and $N=800$. However, we should note that this in turn increases the average delay, thus, there is a clear trade-off. Therefore, the selection of these thresholds should be made by considering the QoS requirements of the V2X communication applications [26].

\section{Throughput and Channel Utilization}

The behavior of the throughput with $N$ is illustrated in Fig. 8. In IEEE 802.11p, we can observe that the throughput increases with $N$ first, which is rather intuitive. However, when more vehicles contend for transmission simultaneously, the delay and the collisions increase, and hence, the throughput starts to decline. Although not seen in the figure, the curve for $S_{M}^{11 p}$ single stream tends to decrease at large $N$ values as well. Due to having no repetitions, MHD has the lowest transmit rate, hence the lowest number of collisions. Thus, the value of $N$ at which the collisions and the delay start dominating the throughput is comparatively large $(N \approx 3500)$ for MHD. The value of $N$ at which the declining stage begins reduces when the data rates increase, which is expected. This can be observed by comparing the single stream operations with the parallel multiple streams, which has more frequent packet transmissions and higher collision probability (see Fig. 7a). The collisions also lead to lower throughput per vehicle when parallel multi-priority data streams are used for communication. Since we consider broadcast packets, the calculated average delay values in this paper only capture the latency from packet generation to packet transmission, and not till successful packet reception. Hence, the probability of collision plays an important role in the performance of the protocols. For example, Fig. 7a clearly exhibits that a small change in collision probability has a huge impact on the overall performance in terms of throughput. We can also observe that the throughput decreases further with the use of adaptive CAM rates. Adaptive CAM reduces the CAM packet generation rate when the channel is congested, which in turn decreases the throughput.

On the other hand, in C-V2X Mode 4, we can observe that the throughput increases with $N$, with a sudden reduction when the vehicle switches from a shorter to a longer selection window size. This is due to the reduction in $P_{t x o}$, which is proportional to $P_{t}^{v 2 x}$. It is interesting to note that the parallel multiple stream scenario of C-V2X Mode 4 exhibits higher throughput compared to the single stream operations, unlike IEEE $802.11 \mathrm{p}$. Thus, it can be noted that C-V2X Mode 4 manages multi-priority data streams in a better manner with regards to throughput, when compared with IEEE 802.11p.

The behavior of the channel utilization with $N$ is illustrated in Fig. 9. The parallel operation of multiple streams depict higher channel utilization for both technologies. This implies that in an actual network, the channel is well utilized by both of these technologies, with C-V2X Mode 4 having a slightly lower utilization, comparatively. We can observe the channel utilization varying with adaptive CAM rates and with selection window sizes. The behavior can be explained using the same reasons provided for the throughput.

\section{CONCLUSIONS}

This paper has presented analytical models for the MAC layer operations of ITS-G5 IEEE 802.11p and C-V2X Mode 4, considering four concurrent multi-priority data streams. Four DTMC-based traffic generators have been used to generate the HPD, DENM, CAM, and MHD packets. The packet generation parameters for each type of data stream have been selected according to ETSI standards. Four device-level queue models have been used to feed the generated multi-priority packets to the DTMCs modeling the MAC layer operations. The steady-state probabilities of the models have been utilized to 


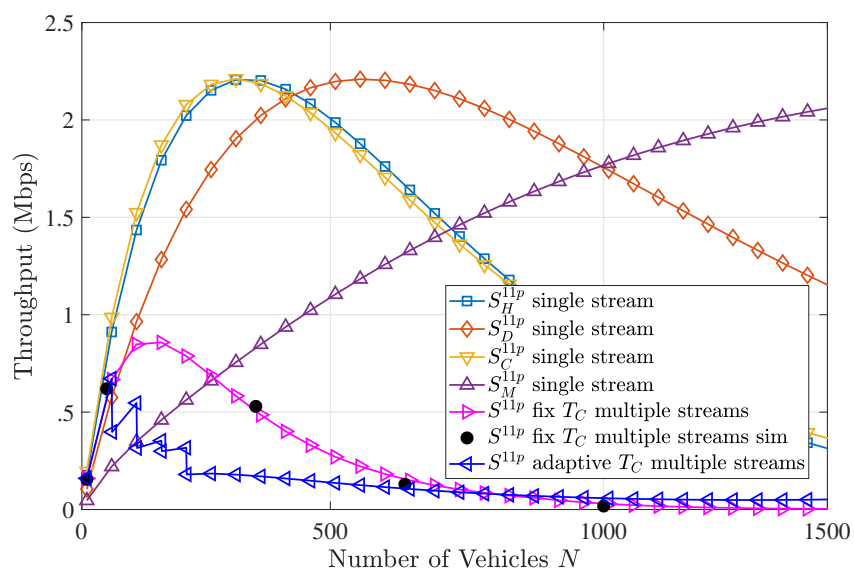

(a) IEEE $802.11 \mathrm{p}$.

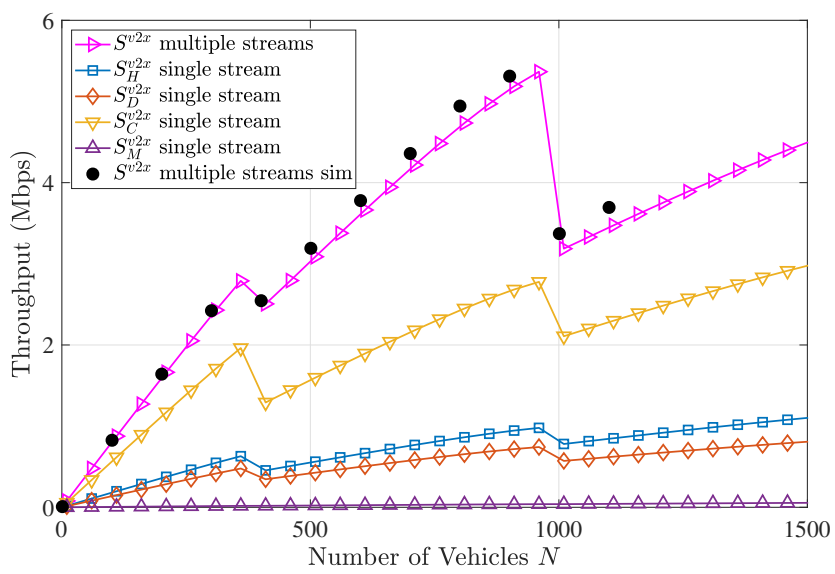

(b) C-V2X Mode 4.

Figure 8: The behavior of the throughput with $N$, where $T_{C}=100 \mathrm{~ms}$.

derive expressions for key performance indicators at the MAC layer, which includes the average delay, collision probability, throughput, and channel utilization. Numerical examples have been presented for further insights. The results have firstly highlighted the importance of considering all four parallel data streams when studying the MAC layer performance of the two technologies. Priority management is implemented successfully in both technologies, but it can be seen that IEEE $802.11 \mathrm{p}$ treats multi-priority data streams more fairly compared to its counterpart, thanks to its inbuilt EDCA mechanism. IEEE $802.11 \mathrm{p}$ is also superior in average delay, but C-V2X Mode 4 exhibits better performance in terms of collision resolution, in turn leading to higher throughput values as well. Further insights have been presented on the possible improvements of both technologies.

\section{APPENDIX A}

\section{The STEAdy-STATE SOlutions FOR THE DTMCs}

\section{A. Generator Models}

For $i \in\{H, D\}$ and $k \in\{11 p, v 2 x\}$, we have $\pi_{i,(t x, 0)}^{k}=\left[T_{i}\left(1-\frac{1}{K_{i}}\right)\left[1-\mathcal{P}_{i}^{k}\left(1-\mathcal{P}_{i}^{k}\right)^{T_{i}-1}\right][1-\right.$ $\left.\left.\left(1-\mathcal{P}_{i}^{k}\right)^{T_{i}-1}\right]^{-1}+1 / K_{i}+\left[K_{i}\left(1-e^{-\lambda_{i} \tilde{T}}\right)\right]^{-1}\right]^{-1}, \pi_{i,(t x, j)}^{k}=$ $\mathcal{P}_{i}^{k}\left[\left(1-\frac{1}{K_{i}}\right) \pi_{i,(t x, 0)}^{k}+\sum_{m=j+1}^{T_{i}-1} \pi_{i,\left(t x^{\prime}, m\right)}^{k}\right]$ for $j \in\left[1, T_{i}-\right.$

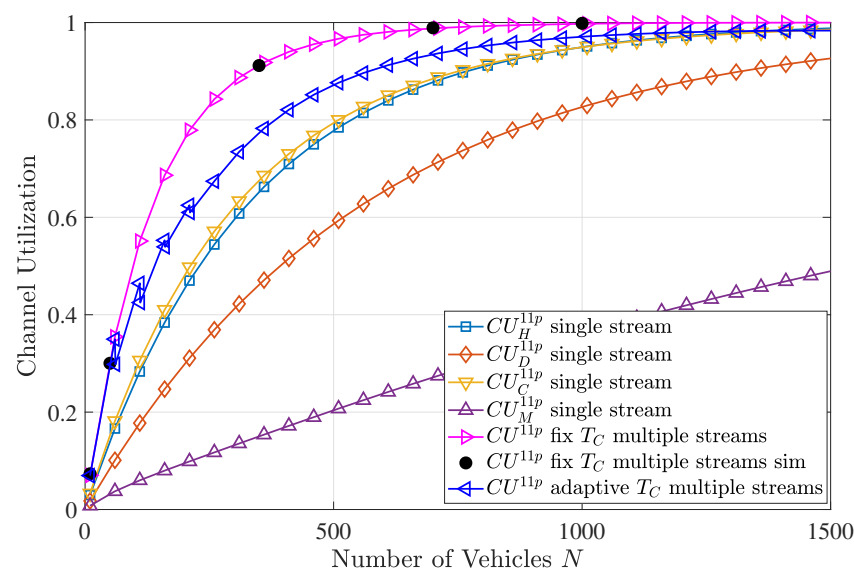

(a) IEEE $802.11 \mathrm{p}$.

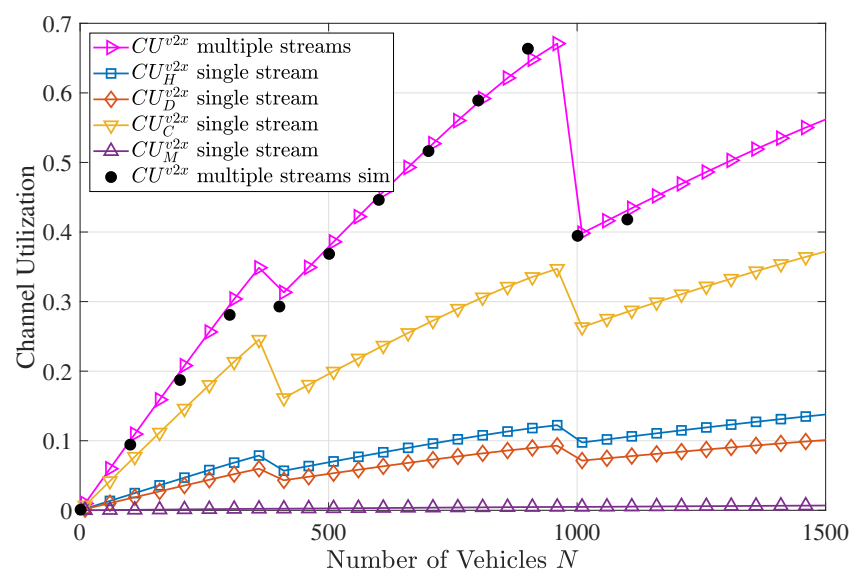

(b) C-V2X Mode 4.

Figure 9: The behavior of the channel utilization with $N$, where $T_{C}=100 \mathrm{~ms}$. $2], \pi_{i,\left(t x, T_{i}-1\right)}^{k}=\pi_{i,(t x, 0)}^{k}\left(1-\frac{1}{K_{i}}\right) \mathcal{P}_{i}^{k}$, and $\pi_{i,\left(t x^{\prime}, j\right)}^{k}=$ $\pi_{i,(t x, 0)}^{k}\left(1-\frac{1}{K_{i}}\right)\left(1-\mathcal{P}_{i}^{k}\right)^{T_{i}-j}\left[1-\left(1-\mathcal{P}_{i}^{k}\right)^{T_{i}-1}\right]^{-1}$ for $j \in\left[0, T_{i}-1\right]$.

For the CAM generator, for $k \in\{11 p, v 2 x\}, \pi_{C,(t x, 0)}^{k}=$ $\left[1-\left(1-\mathcal{P}_{C}^{k}\right)^{T_{C}-1}\right]\left[T_{C}\left[1-\mathcal{P}_{C}^{k}\left(1-\mathcal{P}_{C}^{k}\right)^{T_{C}-1}\right]\right]^{-1}, \pi_{C,(t x, j)}^{k}=$ $\mathcal{P}_{C}^{k}\left(\pi_{C,(t x, 0)}^{k}+\sum_{m=j+1}^{T_{C}-1} \pi_{C,\left(t x^{\prime}, m\right)}^{k}\right)$ for $j \in\left[1, T_{C}-2\right]$, $\pi_{C,\left(t x, T_{C}-1\right)}^{k}=\pi_{C,(t x, 0)}^{k} \mathcal{P}_{C}^{k}$, and $\pi_{C,\left(t x^{\prime}, j\right)}^{k}=\pi_{C,(t x, 0)}^{k}[(1-$ $\left.\left.\mathcal{P}_{C}^{k}\right)^{T_{C}-j}\right]\left[1-\left(1-\mathcal{P}_{C}^{k}\right)^{T_{C}-1}\right]^{-1}$ for $j \in\left[0, T_{C}-1\right]$.

\section{B. IEEE 802.11p ACs Models}

1) Steady-state Solutions for the States Common to All ACs: For $i \in \mathcal{A C}, \pi_{A_{i}^{j}}=\pi_{i d l e_{i}} \Phi_{i}$ for $j=1, \pi_{A_{i}^{j}}=$ $\pi_{\text {idle }_{i}} \Phi_{i}\left(1-\hat{\theta}_{o, i}\right)\left(1-\hat{\theta}_{s, i}\right)^{(j-2)}$ for $j \in\left[2,\left(\Omega_{i}-1\right)\right], \pi_{I_{i}, j}=$ $\pi_{B_{v o}, \vartheta}\left(C_{v o}-j\right) /\left[C_{v o}\left(1-\hat{\theta}_{s, i}\right)\right]$ for $j \in\left[0,\left(C_{v o}-1\right)\right]$, $\pi_{B_{i}, j}=\pi_{\text {Idle }_{i}} \Phi_{i}\left[j \frac{\hat{\theta}_{o, i}}{\vartheta}+\left(1-\hat{\theta}_{o, i}\right)\left[1-\left(1-\hat{\theta}_{s, i}\right)^{\Omega_{i}-1}\right]\right]$, and $\pi_{T_{i}, j}=\pi_{\text {Idle }_{i}} \Phi_{i}$ for $j \in[1, \vartheta]$.

2) Steady-state Solutions for States Specific to $A C_{v o}$ : $\pi_{\Delta_{v o}^{b}, j}=\pi_{B_{v o}, \vartheta}\left(C_{v o}-k\right) \hat{\theta}_{s, v o} /\left[C_{v o}\left(1-\hat{\theta}_{s, v o}\right)\right]$ for $b \in\left[0,\left(C_{v o}-1\right)\right], j \in[1, \vartheta]$. For $j \in\left[1,\left(\Omega_{b k}-8\right)\right], \pi_{b, A_{v o}^{j}}=$ $\pi_{B_{v o}, \vartheta}\left[1+\left(C_{v o}-b-1\right) \hat{\theta}_{s, v o}\right] /\left[C_{v o}\left(1-\hat{\theta}_{s, v o}\right)\right]$ for $b \in$ 
$\left[2,\left(C_{v o}-1\right)\right]$ and $\pi_{b, A_{v o}^{j}}=\pi_{B_{v o}, \vartheta}\left(2-2 \hat{\theta}_{s, v o}+\right.$ $\left.C_{v o} \hat{\theta}_{s, v o}\right) /\left[C_{v o}\left(1-\hat{\theta}_{s, v o}\right)\right]$ for $b=0$.

3) Steady-state Solutions for States Specific to $A C_{v i}$ : For $j \in[1, \vartheta], \pi_{\Delta_{v i}^{b}, j}=\pi_{B_{v i}, \vartheta}\left[\theta_{v o}+\right.$ $\left.\left(C_{v i}-b-\theta_{v o}\right) \hat{\theta}_{s, v i}\right] /\left[C_{v i}\left(1-\theta_{v o}\right)\left(1-\hat{\theta}_{s, v i}\right)\right]$ for $b \in$ $\left[2,\left(C_{v i}-1\right)\right]$ and $\pi_{\Delta_{v i}^{b}, j}=\pi_{B_{v i}, \vartheta}\left[2 \theta_{v o}+\left(C_{v i}-\right.\right.$ $\left.\left.2 \theta_{v o}\right) \hat{\theta}_{s, v i}\right] /\left[C_{v i}\left(1-\theta_{v o}\right)\left(1-\hat{\theta}_{s, v i}\right)\right]$ for $b=0$. For $j \in\left[1,\left(\Omega_{b k}-7\right)\right], \pi_{b, A_{v i}^{j}}=\pi_{B_{v i}, \vartheta}\left[1+\left(C_{v i}-b-\right.\right.$ $\left.1) \hat{\theta}_{s, v i}\right] /\left[C_{v i}\left(1-\hat{\theta}_{s, v i}\right)\left(1-\theta_{v o}\right)\right]$ for $b \in\left[2,\left(C_{v i}-1\right)\right]$ and $\pi_{b, A_{v i}^{j}}=\pi_{B_{v i}, \vartheta}\left(2-2 \hat{\theta}_{s, v i}+C_{v i} \hat{\theta}_{s, v i}\right)\left[C_{v i}\left(1-\hat{\theta}_{s, v i}\right)(1-\right.$ $\left.\left.\theta_{v o}\right)\right]^{2}$ for $b=0$.

4) Steady-state Solutions for States Specific to $A C_{b e}$ : For $j \in[1, \vartheta], G_{1}=\theta_{v o}+\left(1-\theta_{v o}\right)\left[1-\left(1-\theta_{v o}-\theta_{v i}\right)^{3}(1-\right.$ $\left.\left.\hat{\theta}_{s, b e}\right)\right], \pi_{\Delta_{b e}^{b}, j}=\left[G_{1}+\left(C_{b e}-b-1\right) \hat{\theta}_{s, b e}\right] /\left[C_{b e}(1-\right.$ $\left.\left.\theta_{v o}\right)\left(1-\theta_{v o}-\theta_{v i}\right)^{3}\left(1-\hat{\theta}_{s, b e}\right)\right]$ for $b \in\left[2,\left(C_{b e}-1\right)\right]$ and $\pi_{\Delta_{b e}^{b}, j}=\left[2 G_{1}+\left(C_{b e}-2\right) \hat{\theta}_{s, b e}\right] /\left[C_{b e}\left(1-\theta_{v o}\right)(1-\right.$ $\left.\left.\theta_{v o}-\theta_{v i}\right)^{3}\left(1-\hat{\theta}_{s, b e}\right)\right]$ for $b=0$. For $j \in\left[1,\left(\Omega_{b k}-1\right)\right], G_{2}(b)=\pi_{B_{b e}, \vartheta}[1+$ $\left.\left(C_{b e}-b-1\right) \hat{\theta}_{s, b e}\right] /\left[C_{b e}\left(1-\hat{\theta}_{s, b e}\right)\left(1-\theta_{v o}\right)\left(1-\theta_{v o}-\right.\right.$ $\left.\left.\theta_{v i}\right)^{3}\right], f_{1}(j)=1 /\left(1-\theta_{v o}\right)$ for $j \in\left[1,\left(\Omega_{b k}-4\right)\right], f_{1}(j)=$ $\left(1-\theta_{v o}-\theta_{v i}\right)^{b}$ for $j=\left(\Omega_{b k}-3+b\right)$ and $b \in\{0,1,2\}$. $\pi_{b, A_{b e}^{j}}=G_{2}(b) f_{1}(j)\left(1-\theta_{v o}\right)$ for $b \in\left[2, C_{b e}-1\right]$, and $\pi_{b, A_{b e}^{j}}=G_{2}(b)\left[2+\left(C_{b e}-2\right) \hat{\theta}_{s, b e}\right] /\left[1+\left(C_{b e}-b-\right.\right.$ 1) $\left.\hat{\theta}_{s, b e}\right]$ for $b=0$.

5) Steady-state Solutions for States Specific to $A C_{b k}$ : For $j \in[1, \vartheta], G_{3}=\theta_{v o}+\left(1-\theta_{v o}\right)\left[1-\left(1-\theta_{v o}-\right.\right.$ $\left.\left.\theta_{v i}\right)^{3}\left[\left(1-\sum_{l \in \mathcal{A C} \backslash\{b k\}} \theta_{l}\right)^{3}+\left(1-\hat{\theta}_{s, b k}\right)\right]\right], \pi_{\Delta_{b k}^{b}, j}=\left[G_{3}+\right.$ $\left.\left(C_{b k}-b-1\right) \hat{\theta}_{s, b k}\right] /\left[C_{b k}\left(1-\theta_{v o}\left(1-\theta_{v o}-\theta_{v i}\right)^{3}(1-\right.\right.$ $\left.\left.\sum_{l \in \mathcal{A C} \backslash\{b k\}} \theta_{l}\right)^{3}\left(1-\hat{\theta}_{s, b k}\right)\right]$ for $b \in\left[2,\left(C_{b k}-1\right)\right]$ and $\pi_{\Delta_{b k}^{b}, j}=$ $\left[2 G_{3}+\left(C_{b k}-2\right) \hat{\theta}_{s, b k}\right] /\left[C_{b k}\left(1-\theta_{v o}\right)\left(1-\theta_{v o}-\theta_{v i}\right)^{3}(1-\right.$ $\left.\left.\theta_{v o}-\theta_{v i}-\theta_{b e}\right)^{3}\left(1-\hat{\theta}_{s, b k}\right)\right]$ for $b=0$. For $j \in\left[1,\left(\Omega_{b k}-\right.\right.$ $1)], G_{4}(b)=\pi_{B_{b k}, \vartheta}\left[1+\left(C_{b k}-b-1\right) \hat{\theta}_{s, b k}\right] /\left[C_{b k}(1-\right.$ $\left.\left.\hat{\theta}_{s, b k}\right)\left(1-\theta_{v o}\right)\left(1-\theta_{v o}-\theta_{v i}\right)^{3}\left(1-\sum_{l \in \mathcal{A C} \backslash\{b k\}} \theta_{l}\right)^{3}\right], f_{2}(j)=$ $1 /\left(1-\theta_{v o}\right)$ for $j \in\left[1,\left(\Omega_{b k}-7\right)\right], f_{2}(j)=\left(1-\theta_{v o}-\right.$ $\left.\theta_{v i}\right)^{b}$ for $j=\left(\Omega_{b k}-6+b\right)$ and $b \in\{0,1,2,3\}, f_{2}(j)=$ $\left(1-\theta_{v o}-\theta_{v i}\right)^{3}\left(1-\theta_{v o}-\theta_{v i}-\theta_{b e}\right)^{b+1}$ for $j=\left(\Omega_{b k}-\right.$ $2+b)$ and $b \in\{0,1\} \cdot \pi_{b, A_{b k}^{j}}=G_{4}(b) \times f_{2}(j)$ for $b \in$ $\left[2, C_{b k}-1\right]$ and $\pi_{b, A_{b k}^{j}}=f_{2}(j)\left(1-\theta_{v o}\right) G_{4}(b)\left[2+\left(C_{b k}-\right.\right.$ 2) $\left.\hat{\theta}_{s, b k}\right] /\left[1+\left(C_{b k}-b-1\right) \hat{\theta}_{s, b k}\right]$ for $b=0$.

By using the sum of steady-state probabilities, and appropriately substituting the above derived steady-state probabilities, we can obtain $\pi_{\text {idle }_{i}}$ for $i \in \mathcal{A C}$, which can then be used to calculate all steady-state probabilities of interest.

\section{C-V2X Mode 4 Model}

The idle state: $\pi_{\text {Idle }}=\gamma \pi_{w, 0}$, where $\gamma=(1-$ $\left.P_{r k}\right)\left[\left(1 / P_{s c h}\right)-1\right]\left[P_{a r r}^{v 2 x}+P_{q n e}^{v 2 x}\left(1-P_{a r r}\right)\right]^{-1}$. The waiting states $(w, i)$ for $i \in[0, \Gamma-2]: \pi_{w, j}=[1-$
$\left.\frac{j}{(\Gamma-1)}\right]\left[a \pi_{I d l e}^{v 2 x}+\left(1-P_{r k}\right) P_{\text {sch }} \pi_{1,0} P_{q n e}\right]+P_{r k} \pi_{1,0} P_{q n e}$, where $a=\left(P_{a r r}^{v 2 x}+P_{q n e}^{v 2 x}-P_{a r r}^{v 2 x} P_{q n e}^{v 2 x}\right) P_{\text {sch }}$. The state $(i, j)$ : $\pi_{i, j}=\pi_{w, 0}\left(R_{h}-i+1\right) /\left[\left(P_{\text {qne }}^{v 2 x}\right)^{2}\left(1+R_{h}-R_{l}\right)\right]$ for $i \in$ $\left[R_{l}, R_{h}\right], j \in[1, \Gamma-1]$. The state $(i, 0): \pi_{i, 0}=\pi_{w, 0}\left(R_{h}-\right.$ $i+1) /\left[P_{q n e}^{v 2 x}\left(1+R_{h}-R_{l}\right)\right]$ for $i \in\left[R_{l}, R_{h}\right]$. The state $(i, j)$ : $\pi_{i, j}=\pi_{w, 0} / P_{q n e}^{v 2 x}$, for $i \in\left[1, R_{l}-1\right], j \in[0, \Gamma-1]$. Since the sum of probabilities is one, we have $\pi_{w, 0}=[1-\Gamma+\gamma+$ $\left.\left(\frac{\Gamma-2}{2}\right)\left[a \gamma+2 P_{r k}+\left(1-P_{r k}\right) P_{s c h}\right]+\left[\Gamma\left(R_{h}+R_{l}\right) / 2 P_{q n e}^{v 2 x}\right]\right]^{-1}$.

\section{APPENDIX B}

\section{Performance Metric Derivations}

\section{A. Average Delay Derivations: Proof of Lemma 1}

For $A C_{i}$, the cycle time of state $\left(T_{i}, 1\right)$ can be written as aSlotTime $/ \pi_{T_{i}, 1}$, which gives the average time taken to initiate two consecutive transmissions. We modify this cycle time by scaling it by $\left(1-\pi_{\text {idle }_{i}}\right)$, which eliminates the time spent at state $\left(i d l e_{i}\right)$ without any packets to transmit, as this cannot be considered to be a part of the delay. We add $(\vartheta-1)$ aSlotTime to this value to account for the further time spent in transmission similar to [14]. The delay will vary depending on the length of the queue. Since we are dealing with the average delay, we multiply by the resulting delay by the average queue length, which is given by $\sum_{j=0}^{Q^{\max }}(j+1) \pi_{j, l}^{11 p}$, where $\pi_{j, l}^{11 p}$ is the steady state probability of state $j$ of queue $l$. The proof for $d_{a v g, l}^{v 2 x}$ can be obtained by following similar lines as in [14], thus omitted in this paper due to space limitations.

\section{B. Collision Probability Derivations: Proof of Lemma 2}

According to the IEEE $802.11 \mathrm{p}$ standard, a collision occurs when multiple vehicles initiate their transmission simultaneously, or in the case of a self-collision. Firstly, by eliminating the probabilities of no vehicles initiating transmission or exactly one vehicle initiating transmission from the total probability, we get the probability of two or more vehicles initiating their transmission simultaneously. Then, we calculate the self-collision probability by considering a single vehicle, and eliminating the probabilities of no data transmission or exactly one data stream initiating transmission from the total probability. The summation of these two probabilities gives the expression for $P_{c o l}^{11 p}$. The proof for $P_{c o l}^{v 2 x}$ can be obtained by following similar lines as in [14], thus omitted in this paper due to space limitations.

\section{REFERENCES}

[1] ETSI, "Intelligent transport systems (ITS); Vehicular communications; geonetworking; part 4: Geographical addressing and forwarding for point-to-point and point-to-multipoint communications; sub-part 2: Media-dependent functionalities for ITS-G5," Technical Specification (TS) 102 636-4-2, European Telecommunications Standards Institute (ETSI), Oct. 2013. Version 1.1.1.

[2] ETSI, "Intelligent transport systems (ITS); vehicular communications; basic set of applications; part 2: Specification of cooperative awareness basic service," Technical Specification (TS) 102 637-2, European Telecommunications Standards Institute (ETSI), Mar. 2011. Version 1.2.1.

[3] ETSI, "Intelligent transport systems (ITS); vehicular communications; basic set of applications; part 3: Specification of decentralized environmental notification basic service," Technical Specification (TS) 102 637-3, European Telecommunications Standards Institute (ETSI), Sep. 2010. Version 1.1.1. 
[4] IEEE, "IEEE standard for information technology- local and metropolitan area networks- specific requirements- part 11: Wireless LAN medium access control (MAC) and physical layer (PHY) specifications amendment 6: Wireless access in vehicular environments," IEEE Std 802.11p-2010, pp. 1-51, Jul. 2010.

[5] IEEE, "IEEE standard for information technology-telecommunications and information exchange between systems local and metropolitan area networks - specific requirements - part 11: Wireless LAN medium access control (MAC) and physical layer (PHY) specifications," IEEE Std 802.11-2016, pp. 1-3534, Dec. 2016.

[6] 3GPP, "Evolved universal terrestrial radio access (E-UTRA) and evolved universal terrestrial radio access network (E-UTRAN); Overall description; stage 2," Technical Specification (TS) 36.300, 3rd Generation Partnership Project (3GPP), Dec. 2016. Version 14.1.0.

[7] 3GPP, "Evolved universal terrestrial radio access (E-UTRA); Physical layer procedures," Technical Specification (TS) 36.213, 3rd Generation Partnership Project (3GPP), Apr. 2017. Version 14.2.0.

[8] 3GPP, "Evolved universal terrestrial radio access (E-UTRA); Medium access control (MAC) protocol specification," Technical Specification (TS) 36.321, 3rd Generation Partnership Project (3GPP), Jul. 2017. Version 14.3.0.

[9] A. F. M. Shahen Shah, H. Ilhan, and U. Tureli, "Modeling and performance analysis of the IEEE 802.11p MAC for VANETs," in Proc. International Conference on Telecommunications and Signal Processing, Budapest, Hungary, pp. 393-396, Jul. 2019.

[10] F. Kaabi, P. Cataldi, F. Filali, and C. Bonnet, "Performance analysis of IEEE 802.11p control channel," in Proc. International Conference on Mobile Ad-hoc and Sensor Networks, Hangzhou, China, pp. 211-214, Dec. 2010.

[11] C. Han, M. Dianati, R. Tafazolli, R. Kernchen, and X. Shen, "Analytical study of the IEEE 802.11p MAC sublayer in vehicular networks," IEEE Trans. Intell. Transp. Syst., vol. 13, pp. 873-886, Jun. 2012.

[12] J. R. Gallardo, D. Makrakis, and H. T. Mouftah, "Performance analysis of the EDCA medium access mechanism over the control channel of an IEEE 802.11p WAVE vehicular network," in Proc. IEEE International Conference on Communications, Dresden, Germany, pp. 1-6, Jun. 2009

[13] J. Zheng and Q. Wu, "Performance modeling and analysis of the IEEE 802.11p EDCA mechanism for VANET," IEEE Trans. Veh. Technol., vol. 65, pp. 2673-2687, Apr. 2016.

[14] G. P. Wijesiri, J. Haapola, and T. Samarasinghe, "A discrete-time Markov chain based comparison of the MAC layer performance of C-V2X Mode 4 and IEEE 802.11p," IEEE Trans. Commun., vol. 69, no. 4, pp. 25052517, Apr. 2021.

[15] F. Eckermann, M. Kahlert, and C. Wietfeld, "Performance analysis of $\mathrm{C}-\mathrm{V} 2 \mathrm{X}$ Mode 4 communication introducing an open-source C-V2X simulator," in Proc. IEEE Vehicular Technology Conference, Honolulu, USA, pp. 1-5, Nov. 2019.

[16] R. Molina-Masegosa, J. Gozalvez, and M. Sepulcre, "Configuration of the C-V2X Mode 4 sidelink PC5 interface for vehicular communication," in Proc. International Conference on Mobile Ad-Hoc and Sensor Networks, Shenyang, China, pp. 43-48, Dec. 2018.

[17] M. Gonzalez-Martín, M. Sepulcre, R. Molina-Masegosa, and J. Gozalvez, "Analytical models of the performance of C-V2X Mode 4 vehicular communications," IEEE Trans. Veh. Technol., vol. 68, pp. 1155-1166, Feb. 2019.

[18] R. Molina-Masegosa, J. Gozalvez, and M. Sepulcre, "Comparison of IEEE 802.11p and LTE-V2X: An evaluation with periodic and aperiodic messages of constant and variable size," IEEE Access, vol. 8, pp. 121526-121548, Jul. 2020.

[19] G. P. Wijesiri, J. Haapola, and T. Samarasinghe, "A Markov perspective on C-V2X Mode 4," in Proc. IEEE Vehicular Technology Conference, Hawaii, USA, pp. 1-6, Sep. 2019.

[20] G. Naik, B. Choudhury, and J. Park, "IEEE 802.11bd 5G NR V2X: Evolution of radio access technologies for V2X communications," IEEE Access, vol. 7, pp. 70169-70184, May 2019.

[21] A. Bazzi, G. Cecchini, M. Menarini, B. M. Masini, and A. Zanella, "Survey and perspectives of vehicular Wi-Fi versus sidelink cellularV2X in the 5G era," Future Internet, vol. 11, p. 122, May 2019.

[22] J. Norris, Markov Chains. Cambridge University Press, 1998.

[23] C. D. Manning, P. Raghavan, and H. Schutze, Introduction to Information Retrieval. Cambridge University Press, 2008.

[24] F. Romeo, C. Campolo, A. Molinaro, and A. O. Berthet, "DENM repetitions to enhance reliability of the autonomous mode in NR V2X sidelink," in Proc. IEEE Vehicular Technology Conference, Antwerp, Belgium, pp. 1-5, May 2020.

[25] G. P. Wijesiri, J. Haapola, and T. Samarasinghe, "The effect of multiple access categories on the MAC layer performance of IEEE 802.11p," in
Proc. IEEE Global Communication Conference, Taipei, Taiwan, Dec. 2020.

[26] ETSI, "Intelligent transport systems (ITS); vehicular communications; Basic Set of Applications; Definitions," Technical Report (TR) 102638 , European Telecommunications Standards Institute (ETSI), Jun. 2009. Version 1.1.1.

[27] ETSI, "Intelligent transport systems (ITS); Access layer specification for intelligent transport system operating in the $5 \mathrm{GHz}$ frequency band," European Standard telecommunications series (EN) 302 663, European Telecommunications Standards Institute (ETSI), May 2013. Version 1.2.1.

[28] A. Alsuhaim, "NS3 example project," Nov. 2020.

[29] Y. Y. Nasrallah, I. Al-Anbagi, and H. T. Mouftah, "A realistic analytical model of IEEE 802.11p for wireless access in vehicular networks," in Proc. International Conference on Connected Vehicles and Expo, Vienna, Austria, pp. 1029-1034, Nov. 2014.

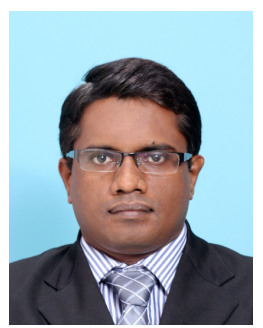

Geeth P. Wijesiri N. B. A. (M'18) was born in Galle, Sri Lanka, in 1982. He received the B.Sc. Engineering (First Class Honors) degree from the Department of Electrical and Information Engineering, University of Ruhuna, Sri Lanka, in 2008 and worked as a Senior Engineer at Lanka Bell Ltd, Sri Lanka for five years $(2008$ - 2013). Then he received his M.Sc. Degree in Information and Communication Technology from the University of Agder, Norway, in 2016. Then, he joined the Department of Electrical and Information Engineering, University of Ruhuna, Sri Lanka, as a lecture in 2016 (currently on study leave). Since May 2018, he has been with the Department of Electronic and Telecommunication Engineering, University of Moratuwa, Sri Lanka, where he is a Ph.D. student. His research interests are in computer networks, vehicular communications, and wireless networks.

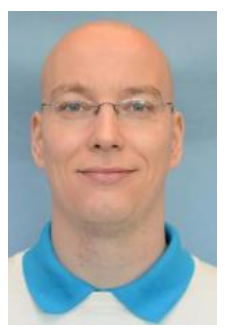

Jussi Haapola, adjunct professor, received his M.Sc. and $\mathrm{Ph} . \mathrm{D}$. degrees from the University of Oulu, Finland, in 2002 from Physical Sciences and in 2010 from Telecommunications Engineering, respectively. He joined the Centre for Wireless Communications at the University of Oulu in 2001, where he currently works as a Senior Research Fellow on heterogeneous IoT and cellular systems for vertical applications. In 2011 he made a Post-doctoral Fellow visit in Tohoku University, Sendai, Japan and between 2012 and 2015 he also worked for University of Oulu Research Institute Japan - CWC-Nippon Ltd as Deputy manager. He is currently the coordinator of EC H2020 project 5G!Drones (No. 857031).

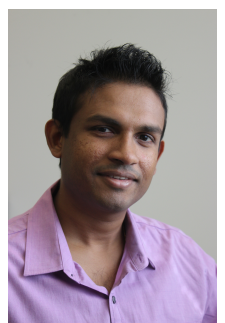

Tharaka Samarasinghe (S'11-M'13) was born in Colombo, Sri Lanka, in 1984. He received the B.Sc. degree in engineering from the Department of Electronic and Telecommunication Engineering, University of Moratuwa, Sri Lanka, in 2008, where he received the award for the most outstanding undergraduate upon graduation. He received the Ph.D. degree from the Department of Electrical and Electronic Engineering, University of Melbourne, Australia, in 2012. He was a Research Fellow at the Department of Electrical and Computer Systems Engineering, Monash University, Australia, from 2012 to 2014. He has been with the Department of Electronic and Telecommunication Engineering, University of Moratuwa, Sri Lanka, since January 2015, where he is a Senior Lecturer. He is also an honorary fellow at the Department of Electrical and Electronic Engineering, University of Melbourne, Australia. His research interests are in communications theory, information theory, and wireless networks. 